\title{
Base norms and discrimination of generalized quantum channels
}

\author{
A. Jenčová \\ Mathematical Institute, Slovak Academy of Sciences \\ Štefánikova 49, Bratislava, Slovakia
}

\begin{abstract}
We introduce and study norms in the space of hermitian matrices, obtained from base norms in positively generated subspaces. These norms are closely related to discrimination of so-called generalized quantum channels, including quantum states, channels and networks. We further introduce generalized quantum decision problems and show that the maximal average payoff of decision procedures is again given by these norms. We also study optimality of decision procedures, in particular, we obtain a necessary and sufficient condition under which an optimal 1-tester for dicrimination of quantum channels exists, such that the input state is maximally entangled.
\end{abstract}

\section{Introduction and preliminaries}

It is well known that in the problem of discrimination of quantum states, the best possible distinguishability of two states $\rho_{0}$ and $\rho_{1}$ is given by the trace norm $\left\|\rho_{0}-\rho_{1}\right\|_{1},[10,11$. The set of states forms a base of the convex cone of positive operators and the restriction of the trace norm to hermitian operators is the corresponding base norm. Similarly, it was shown in [21 that more general distinguishability measures, obtained by specification of the allowed measurements e.g. for bipartite states, are obtained from base norms associated with more general positive cones. This correspondence is related to duality of the base norm and the order unit norm, with respect to a given positive cone.

In a similar problem for quantum channels, and recently also quantum networks, the diamond norm $\|\cdot\|_{\diamond}$ for channels [15], resp. the strategy $N$-norm $\|\cdot\|_{N \diamond}$, 9, 3] for networks is obtained. Via the Choi isomorphism, quantum networks are represented by certain positive operators on the tensor product of the input and output spaces, so-called $N$-combs [2, 4, see also 8 . The set of $\mathrm{N}$-combs is the intersection of the multipartite state space by a positively generated subspace of the real vector space of hermitian operators. Since this subspace inherits the order structure and the set of $N$-combs forms a base of its positive cone, it is natural to expect that the distinguishability norm $\|\cdot\|_{N \diamond}$ is in fact the corresponding base norm. 
Motivated by this question, we study positively generated subspaces of the space of hermitian operators $B_{h}(\mathcal{H})$ acting on a finite dimensional Hilbert space $\mathcal{H}$. For a given base $B$ of the positive cone, we define a distinguishability measure in terms of tests that are defined as affine maps $B \rightarrow[0,1]$ and show that this measure is given by the base norm, this, in fact, is easy to see for any finite dimensional ordered vector space. We then study a natural extension of this norm to $B_{h}(\mathcal{H})$ and its dual norm. An example of such a base is the set of Choi matrices of so-called generalized channels, this contains the set of $N$-combs as a special case. For $N$-combs, the obtained norm coincides with $\|\cdot\|_{N \diamond}$ and we recover some of the results of [9] concerning the dual norm. Moreover, we find a suitabe expression for this norm, closely related to the definition of $\|\cdot\|_{\diamond}$.

It the last section, we introduce generalized quantum decision problems with respect to a base $B$. We show that maximal average payoff (or minimal average loss) of generalized decision procedures is again given by a base norm. We find optimality conditions for generalized decision procedures, in particular, for quantum measurements and testers. In the case of multiple hypothesis testing for states, we get the results obtained previously in [18, 17, 12. In the case of discrimination of quantum channels, we find a necessary and sufficient condition for existence of an optimal tester such that the input state is maximally entangled.

The rest of the present section contains some basic definitions and preliminary results on discrimination of quantum devices, as well as convex cones, bases and base norms.

\subsection{Discrimination of quantum states, channels and net- works}

Let $\mathcal{H}$ be a finite dimensional Hilbert space and let $B(\mathcal{H})$ be the set of bounded operators on $\mathcal{H}$. We denote by $B_{h}(\mathcal{H})$ the set of self-adjoint operators, $B(\mathcal{H})^{+}$ the cone of positive operators and $\mathfrak{S}(\mathcal{H}):=\{\rho \geq 0, \operatorname{Tr} \rho=1\}$ the set of states in $B(\mathcal{H})$. Let $\mathcal{K}$ be another finite dimensional Hilbert space. It is well known that $B(\mathcal{K} \otimes \mathcal{H})$ corresponds to the set of all linear maps $B(\mathcal{H}) \rightarrow B(\mathcal{K})$, via the Choi representation:

$$
X_{\Phi}=\left(\Phi \otimes i d_{\mathcal{H}}\right)(\Psi), \quad \Phi_{X}(a)=\operatorname{Tr}_{\mathcal{H}}\left[\left(I_{\mathcal{K}} \otimes a^{\top}\right) X\right]
$$

here $\Psi=|\psi\rangle\langle\psi|$ and $|\psi\rangle=\sum_{i}|i\rangle \otimes|i\rangle$ for an $\operatorname{ONB}\{|i\rangle, i=1, \ldots, \operatorname{dim}(\mathcal{H})\}$ in $\mathcal{H}, a^{\top}$ denotes transpose of $a$. In this correspondence, $B(\mathcal{K} \otimes \mathcal{H})^{+}$is identified with the set of completely positive maps and $B_{h}(\mathcal{K} \otimes \mathcal{H})$ with hermitian maps, that is, maps satisfying $\Phi\left(a^{*}\right)=\Phi(a)^{*}$.

Consider the problem of quantum state discrimination: suppose the quantum system represented by $\mathcal{H}$ is known to be in one of two given states $\rho_{0}$ or $\rho_{1}$ and the task is to decide which of them is the true state. This is done by using a test, that is a binary positive operator valued measure (POVM). This is given by an operator $0 \leq M \leq I$, with the interpretation that $\operatorname{Tr} M \rho$ is the probability of deciding for $\rho_{0}$ if the true value of the state is $\rho$. Equivalently, a test can be defined as an affine map $\mathfrak{S}(\mathcal{H}) \rightarrow[0,1]$. 


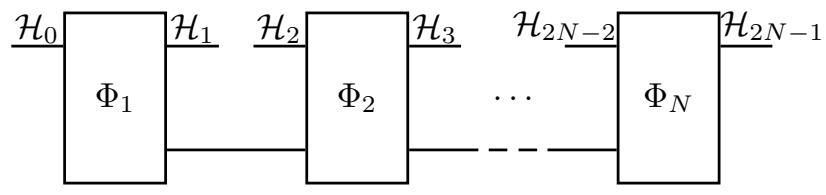

Figure 1: A deterministic quantum $N$-comb

Given an a priori probability $0 \leq \lambda \leq 1$ that the true state is $\rho_{0}$, we need to minimize the average probability of error over all tests, that is to find the value of

$$
\Pi_{\lambda}\left(\rho_{0}, \rho_{1}\right):=\min _{0 \leq M \leq I} \lambda \operatorname{Tr}(I-M) \rho_{0}+(1-\lambda) \operatorname{Tr} M \rho_{1},
$$

this is the minimum Bayes error probability. Then [10, 11]

$$
\Pi_{\lambda}\left(\rho_{0}, \rho_{1}\right)=\frac{1}{2}-\frac{1}{2}\left\|\lambda \rho_{0}-(1-\lambda) \rho_{1}\right\|_{1},
$$

where $\|a\|_{1}:=\operatorname{Tr}|a|, a \in B(\mathcal{H})$ is the trace norm.

Let now $\mathcal{H}$ and $\mathcal{K}$ be two finite dimensional Hilbert spaces and consider the problem of discrimination of channels. Here we have to decide between two channels $\Phi_{0}$ and $\Phi_{1}$ and this time the tests are given by binary quantum 1testers [3, or PPOVMs 22, which are positive operators $T \in B(\mathcal{K} \otimes \mathcal{H})^{+}$, such that $T \leq I_{\mathcal{K}} \otimes \sigma$ for some $\sigma \in \mathfrak{S}(\mathcal{H})$. These correspond to triples $\left(\mathcal{H}_{A}, \rho, M\right)$, where $\mathcal{H}_{A}$ is an ancillary Hilbert space, $\rho \in \mathfrak{S}\left(\mathcal{H} \otimes \mathcal{H}_{A}\right)$ and $0 \leq M \leq I$, $M \in B\left(\mathcal{K} \otimes \mathcal{H}_{A}\right)$. The probability of choosing $\Phi_{0}$ if the true value is $\Phi$ for a tester $T$ is given by

$$
p(T, \Phi):=\operatorname{Tr} T X_{\Phi}=\operatorname{Tr} M\left(\Phi \otimes i d_{A}\right)(\rho) .
$$

The minimum Bayes error probability is now

$\Pi_{\lambda}^{1}\left(\Phi_{0}, \Phi_{1}\right):=\min _{T} \lambda\left(1-p\left(T, \Phi_{0}\right)\right)+(1-\lambda) p(T, \Phi)=\frac{1}{2}-\frac{1}{2}\left\|\lambda \Phi_{0}-(1-\lambda) \Phi_{1}\right\|_{\diamond}$

where the diamond norm $\|\Phi\|_{\diamond}$ for a hermitian map $\Phi$ is defined as [15, 20.

$$
\begin{aligned}
\|\Phi\|_{\diamond} & =\sup _{\operatorname{dim}\left(\mathcal{L}^{\prime}\right)<\infty} \sup _{\rho \in \mathfrak{S}\left(\mathcal{H} \otimes \mathcal{L}^{\prime}\right)}\left\|\Phi \otimes i d_{\mathcal{L}^{\prime}}(\rho)\right\|_{1} \\
& =\sup _{\rho \in \mathfrak{S}(\mathcal{H} \otimes \mathcal{L})}\left\|\Phi \otimes i d_{\mathcal{L}}(\rho)\right\|_{1}, \quad \operatorname{dim}(\mathcal{L})=\operatorname{dim}(\mathcal{H})
\end{aligned}
$$

By duality, this norm is related to the $c b$-norm for completely bounded linear maps, see [17].

Let now $\left\{\mathcal{H}_{0}, \mathcal{H}_{1}, \ldots, \mathcal{H}_{2 N-1}\right\}$ be finite dimensional Hilbert spaces. Consider a sequence of channels $\Phi_{i}: B\left(\mathcal{H}_{2 i-2} \otimes \mathcal{H}_{A}\right) \rightarrow B\left(\mathcal{H}_{2 i-1} \otimes \mathcal{H}_{A}\right), i=1, \ldots, N$, connected by the ancilla $\mathcal{H}_{A}$ as indicated on fig. 1 (the first and last ancilla is traced out). This defines a channel $\Phi: B\left(\mathcal{H}_{0} \otimes \mathcal{H}_{2} \otimes \cdots \otimes \mathcal{H}_{2 N-2}\right) \rightarrow$ 


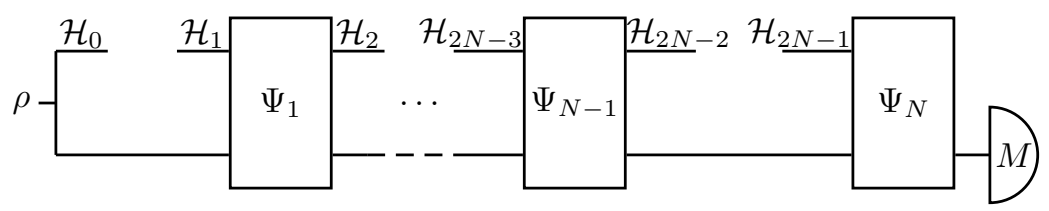

Figure 2: A quantum $N$-tester

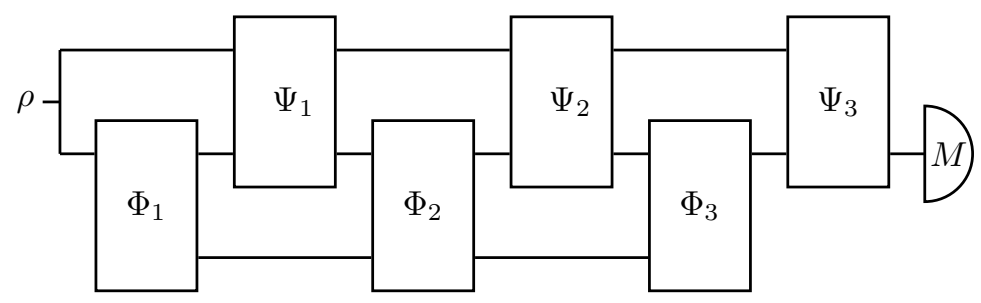

Figure 3: A 3-tester applied to a 3-comb

$B\left(\mathcal{H}_{1} \otimes \mathcal{H}_{3} \otimes \cdots \otimes \mathcal{H}_{2 N-1}\right)$, such channels describe quantum networks. The channels $\Phi_{1}, \ldots, \Phi_{N}$ are not unique, in fact, these can always be supposed to be isometries. A (deterministic) quantum $N$-comb is defined as the Choi matrix $X_{\Phi}$ of such a channel, see 4 for more about quantum networks and $N$-combs. The same definition, called a (non-measuring) quantum $N$-round strategy, was also introduced in [8]. A (non-measuring) quantum $N$-round co-strategy can be defined as an $(N+1)$-strategy for the sequence of spaces $\left\{\mathbb{C}, \mathcal{H}_{0}, \ldots, \mathcal{H}_{2 N-1}, \mathbb{C}\right\}$.

The tests for discrimination of two networks $\Phi^{0}$ and $\Phi^{1}$ are given by quantum $N$-testers, which are obtained by an $(N+1)$-comb such that the first channel has 1-dimensional input space (hence is a state) and a (binary) POVM is applied to the ancilla [4, 3, see figs. 2, 3. This can be represented by a pair $\left(T_{0}, T_{1}\right)$ of positive operators, such that $T_{0}+T_{1}$ is an $(N+1)$-round co-strategy, 4, 8, 9,

The minimal Bayes error probability now has the form

$$
\Pi_{\lambda}^{N}\left(\Phi^{0}, \Phi^{1}\right)=\frac{1}{2}-\frac{1}{2}\left\|\lambda \Phi^{0}-(1-\lambda) \Phi^{1}\right\|_{N \diamond}
$$

where for any hermitian $\Phi$,

$$
\begin{aligned}
\|\Phi\|_{N \diamond} & \left.=\sup _{T} \|\left(T_{0}+T_{1}\right)^{1 / 2} X_{\Phi}\left(T_{0}+T_{1}\right)^{1 / 2}\right] \|_{1}, \\
& =\sup _{T} \operatorname{Tr} X_{\Phi}\left(T_{0}-T_{1}\right), \quad[9
\end{aligned}
$$

where the supremum is taken over all $N$-testers. The dual norm was also obtained in [9], as

$$
\|\Phi\|_{N \diamond}^{*}=\sup _{S} \operatorname{Tr} X_{\Phi}\left(S_{1}-S_{0}\right),
$$


where the supremum is taken over the set of pairs of positive operators such that $S_{0}+S_{1}$ is an $N$-round strategy ( $N$-comb).

\subsection{Convex cones, bases and base norms}

Let $\mathcal{V}$ be a finite dimensional real vector space and let $\mathcal{V}^{*}$ be the dual space, with duality $\langle\cdot, \cdot\rangle$. A subset $Q \subset \mathcal{V}$ is a convex cone if $\lambda q_{1}+\mu q_{2} \in Q$ whenever $q_{1}, q_{2} \in Q$ and $\lambda, \mu \geq 0$. The cone is pointed if $Q \cap-Q=\{0\}$ and generating if $\mathcal{V}=Q-Q$. Closed pointed convex cones are in one-to-one correspondence with partial orders in $\mathcal{V}$, by $x \leq_{Q} y \Longleftrightarrow y-x \in Q$.

The dual cone of $Q$ is defined as

$$
Q^{*}=\left\{f \in \mathcal{V}^{*},\langle f, q\rangle \geq 0, q \in Q\right\}
$$

This is a closed convex cone and $Q^{* *}=Q$ if $Q$ is closed. Moreover, a closed convex cone $Q$ is pointed if and only if $Q^{*}$ is generating. A closed pointed generating convex cone is called a proper cone.

A base of the proper cone $Q$ is a compact convex subset $B \subset Q$, such that each nonzero element $q \in Q$ has a unique representation in the form $q=\lambda b$ with $b \in B$ and $\lambda>0$. It is clear that any base generates the cone $Q$, in the sense that $Q=\bigcup_{\lambda \geq 0} \lambda B$. Then any element $v \in \mathcal{V}$ can be written as $v=\lambda b_{1}-\mu b_{2}$, $\lambda, \mu \geq 0, b_{1}, b_{2} \in B$.

For any base $B$, the map $Q \ni q=\lambda b \mapsto \lambda$ extends uniquely to a linear functional $e_{B} \in Q^{*}$ and we have $B=\left\{q \in Q,\left\langle e_{B}, q\right\rangle=1\right\}$.

Lemma 1. Let $f \in Q^{*}$. Then $f \in \operatorname{int}\left(Q^{*}\right)$ if and only if

$$
B_{f}:=\{q \in Q,\langle f, q\rangle=1\}
$$

is a base of $Q$.

Proof. It is quite clear that $B_{f}$ is a base of $Q$ if and only if $\langle f, q\rangle>0$ for any nonzero $q \in Q$. By [19, Theorem 11.6], this is equivalent with $f \in \operatorname{int}\left(Q^{*}\right)$.

Let $\leq$ denote the order in $\mathcal{V}$ given by $Q$. An element $e \in \mathcal{V}$ is an order unit in $\mathcal{V}$ if for any $v \in \mathcal{V}$, there is some $r>0$ such that $r e \geq v$. It is easy to see that $e$ is an order unit if and only if $e \in \operatorname{int}(Q)$. Consequently,

Corollary 1. Any base $B$ of $Q$ defines an order unit $e_{B}$ in $\mathcal{V}^{*}$ and, conversely, any order unit e in $\mathcal{V}^{*}$ defines a base $B_{e}$ of $Q$. We have $e_{B_{e}}=e$ and $B_{e_{B}}=B$.

Let $B$ be a base of $Q$. The corresponding base norm in $\mathcal{V}$ is defined by

$$
\|v\|_{B}=\inf \left\{\lambda+\mu, v=\lambda b_{1}-\mu b_{2}, \lambda, \mu \geq 0, b_{1}, b_{2} \in B\right\}
$$

It is clear that $\|q\|_{B}=\left\langle e_{B}, q\right\rangle$ for all $q \in Q$. Let $\mathcal{V}_{1}$ be the unit ball of $\|\cdot\|_{B}$ in $\mathcal{V}$, then

$$
\mathcal{V}_{1}=\left\{\lambda b_{1}-\mu b_{2}, b_{1}, b_{2} \in B, \lambda, \mu \geq 0, \lambda+\mu=1\right\}=c o(B \cup-B)
$$


Let $\|\cdot\|_{B}^{*}$ be the dual norm in $\mathcal{V}^{*}$, then the unit ball $\mathcal{V}_{1}^{*}$ for $\|\cdot\|_{B}^{*}$ is given by

$$
\mathcal{V}_{1}^{*}=\mathcal{V}_{1}^{\circ}=(\operatorname{co}(B \cup-B))^{\circ}=(B \cup-B)^{\circ}=B^{\circ} \cap(-B)^{\circ}
$$

where $A^{\circ}:=\left\{f \in \mathcal{V}^{*},\langle f, a\rangle \leq 1, a \in A\right\}$ is the polar of $A \subset \mathcal{V}$, see 19. We have

$$
\mathcal{V}_{1}^{*}=\left\{f \in \mathcal{V}^{*},-1 \leq\langle f, b\rangle \leq 1, b \in B\right\}=\left\{f \in \mathcal{V}^{*},-e_{B} \leq_{Q^{*}} f \leq_{Q^{*}} e_{B}\right\}
$$

where $e_{B}$ is the order unit. Hence the dual norm is given by

$$
\|f\|_{B}^{*}=\inf \left\{\lambda>0,-\lambda e_{B} \leq_{Q^{*}} f \leq_{Q^{*}} \lambda e_{B}\right\}=:\|f\|_{e_{B}}
$$

In general, if $e$ is an order unit, then $\|\cdot\|_{e}$ defines a norm called the order unit norm in $\mathcal{V}^{*}$.

Since $\|\cdot\|_{B}$ is the dual norm for $\|\cdot\|_{e_{B}}$, we get for $v \in \mathcal{V}$,

$$
\|v\|_{B}=\|v\|_{e_{B}}^{*}=\sup _{-e_{B} \leq_{Q^{*}} f \leq_{Q^{*}} e_{B}}\langle f, v\rangle=2 \sup _{f \in Q^{*}, f \leq_{Q^{*}} e_{B}}\langle f, v\rangle-\left\langle e_{B}, v\right\rangle
$$

where the last equality follows by replacing $f$ by $\frac{1}{2}\left(f+e_{B}\right)$.

Example 1. Let $\mathcal{V}=B_{h}(\mathcal{H})$ be the real vector space of self-adjoint elements in $B(\mathcal{H})$ and let $Q=B(\mathcal{H})^{+}$. We identify $\mathcal{V}^{*}$ with $\mathcal{V}$, with duality $\langle a, b\rangle=\operatorname{Tr} a b$, then $Q$ is a self-dual proper cone and $B=\mathfrak{S}(\mathcal{H})$ is a base of $Q$, with $e_{B}=I$. The order unit norm $\|\cdot\|_{I}$ is the operator norm $\|\cdot\|$ in $B(\mathcal{H})$ and its dual $\|\cdot\|_{B}$ is the trace norm $\|\cdot\|_{1}$.

We will finish this section by showing that the base norm is naturally related to a distinguishability measure for elements of the base. By analogy with the set of quantum states, let us define a test on a base $B$ as an affine map $\mathbf{t}: B \rightarrow[0,1]$. It is easy to see that there is a one-to-one correspondence between tests on $B$ and elements $f \leq_{Q^{*}} e_{B}$ in $Q^{*}$. Let $b_{0}, b_{1}$ be two elements of $B$ and let us interpret the value $\mathbf{t}(b)=\langle f, b\rangle$ as the probability of choosing $b_{0}$ if the "true value" is $b$. Then $\left\langle f, b_{1}\right\rangle$ and $1-\left\langle f, b_{0}\right\rangle$ are probabilities of making an error. Let $\lambda \geq 0$, then we define the minimal average error probability as

$$
\Pi_{\lambda}^{B}\left(b_{0}, b_{1}\right):=\min _{0 \leq_{Q^{*}} f \leq_{Q^{*} e_{B}}} \lambda\left(1-\left\langle f, b_{0}\right\rangle\right)+(1-\lambda)\left\langle f, b_{1}\right\rangle
$$

We obtain by (4) that

$$
\begin{aligned}
\Pi_{\lambda}^{B}\left(b_{0}, b_{1}\right) & =\lambda-\max _{0 \leq Q^{*} f \leq Q^{*} e_{B}}\left\langle f, \lambda b_{0}-(1-\lambda) b_{1}\right\rangle \\
& =\frac{1}{2}\left(1-\left\|\lambda b_{0}-(1-\lambda) b_{1}\right\|_{B}\right) .
\end{aligned}
$$

\section{Base norms on subspaces of $B_{h}(\mathcal{H})$}

We now put $\mathcal{V}=B_{h}(\mathcal{H})$, with the self-dual proper cone $B(\mathcal{H})^{+}$as in Example 1. We will describe all possible bases of this cone. 
It is clear that $\operatorname{int}\left(B(\mathcal{H})^{+}\right)$is the set of positive definite elements, hence these are the order units in $B_{h}(\mathcal{H})$. By Corollary 1 , there is a one-to-one correspondence between positive definite elements $b$ and bases of $B(\mathcal{H})^{+}$, given by

$$
b \leftrightarrow S_{b}:=\left\{a \in B(\mathcal{H})^{+}, \operatorname{Tr} a b=1\right\}=B(\mathcal{H})^{+} \cap \mathcal{T}_{b},
$$

where $\mathcal{T}_{b}=\left\{x \in B_{h}(\mathcal{H}), \operatorname{Tr} x b=1\right\}$. For $b \in \operatorname{int}\left(B(\mathcal{H})^{+}\right)$, we have by (4) and Example 1 that the corresponding base norm is

$$
\|x\|_{S_{b}}=\sup _{-b \leq a \leq b} \operatorname{Tr} a x=\sup _{-I \leq a \leq I} \operatorname{Tr} a b^{1 / 2} x b^{1 / 2}=\left\|b^{1 / 2} x b^{1 / 2}\right\|_{1}
$$

and the dual order unit norm is

$$
\|x\|_{b}=\inf \{\lambda>0,-\lambda b \leq x \leq \lambda b\}=\left\|b^{-1 / 2} x b^{-1 / 2}\right\| .
$$

If $b \in B(\mathcal{H})^{+}$is any element, we define

$$
\left\|b^{-1 / 2} x b^{-1 / 2}\right\|:=\lim _{\varepsilon \rightarrow 0^{+}}\left\|(b+\varepsilon)^{-1 / 2} x(b+\varepsilon)^{-1 / 2}\right\|
$$

Note that the expression on the RHS is bounded for all $\varepsilon>0$ if and only if $\operatorname{supp}(x) \leq \operatorname{supp}(b)$ and in this case the norm on the LHS is defined by restriction to the support of $b$. Otherwise, the limit is infinite. Moreover, for any elements $a, b \in B(\mathcal{H})^{+}$, we define

$$
D_{\max }(a \| b):=\log \inf \{\lambda>0, a \leq \lambda b\}=\inf \left\{\gamma>0, a \leq 2^{\gamma} b\right\}
$$

For a pair of states $\rho$ and $\sigma, D_{\max }(\rho \| \sigma)$ is the max-relative entropy of $\rho$ and $\sigma$, introduced in $\left[6.1\right.$ If $b \in \operatorname{int}\left(B(\mathcal{H})^{+}\right)$, then

$$
D_{\max }(a \| b)=\log \left(\|a\|_{b}\right)
$$

In general, if $\operatorname{supp}(a) \leq \operatorname{supp}(b)$, then we may restrict to the support of $b$ and with this restriction $D_{\max }(a \| b)=\log \left(\|a\|_{b}\right)$, otherwise $D_{\max }(a \| b)=\infty$.

\subsection{Sections of a base of $B(\mathcal{H})^{+}$}

Let $J \subset B_{h}(\mathcal{H})$ be a subspace and let $Q=J \cap B(\mathcal{H})^{+}$be the convex cone of positive elements in $J$. It is obvious that $Q$ is closed and pointed. We will suppose that $J$ is positively generated, then $J=Q-Q$ and $Q$ is a proper cone in $J$. Let $b \in Q$ be such that $\operatorname{supp} a \leq \operatorname{supp} b=: p$ for all $a \in Q$, then $J \subseteq B_{h}(p \mathcal{H})$ and by restricting to $B_{h}(p \mathcal{H})$, we may suppose that $b$ is positive definite. Conversely, if $J$ contains a positive definite element, then $J$ is positively generated.

Let $J^{\perp}=\left\{y \in B_{h}(\mathcal{H}), \operatorname{Tr} x y=0, x \in J\right\}$, let $\left.B_{h}(\mathcal{H})\right|_{J^{\perp}}$ be the quotient space and let $\pi:\left.B_{h}(\mathcal{H}) \rightarrow B_{h}(\mathcal{H})\right|_{J^{\perp}}$ be the quotient map $a \mapsto a+J^{\perp}$. We may identify the dual space $J^{*}$ with $\left.B_{h}(\mathcal{H})\right|_{J^{\perp}}$, with duality

$$
\langle x, \pi(a)\rangle=\operatorname{Tr} x a, \quad x \in J, a \in B_{h}(\mathcal{H}) .
$$

\footnotetext{
${ }^{1}$ Note that $D_{\max }$ was denoted by $D_{\infty}$ in 18 .
} 
It was shown in [13] that the dual cone of $Q$ is $Q^{*}=\pi\left(B(\mathcal{H})^{+}\right)$, moreover, since $\pi$ is a linear map, we have $\operatorname{int}\left(Q^{*}\right)=\operatorname{int}\left(\pi\left(B(\mathcal{H})^{+}\right)\right)=\pi\left(\operatorname{int}\left(B(\mathcal{H})^{+}\right)\right)$by [19]. In other words, any element $f \in Q^{*}$ has the form

$$
f(x)=\operatorname{Tr} a x, \quad x \in J
$$

for some (in general non-unique) element $a \in B(\mathcal{H})^{+}$and $f$ is an order unit in $J^{*}$ if and only if $a$ may be chosen positive definite. Now we can use Corollary 1 to describe all bases of $Q$.

Lemma 2. A subset $B \subset Q$ is a base of $Q$ if and only if $B=J \cap S_{\tilde{b}}$, where $\tilde{b} \in \operatorname{int}\left(B(\mathcal{H})^{+}\right)$. In this case, $\pi(\tilde{b})=e_{B}$.

Proof. Let $B$ be a base of $Q$. Since $e_{B} \in \operatorname{int}\left(Q^{*}\right)$, there is some $\tilde{b} \in \operatorname{int}\left(B(\mathcal{H})^{+}\right)$ such that $e_{B}=\pi(\tilde{b})$ and

$$
\left.B=\left\{q \in Q, \operatorname{Tr} q \tilde{b}=\left\langle e_{B}, q\right\rangle=1\right\rangle\right\}=Q \cap \mathcal{T}_{\tilde{b}}=J \cap S_{\tilde{b}}
$$

(see (5) ). Conversely, it is quite clear that $B=J \cap S_{\tilde{b}}$ is a base of $Q$ and $e_{B}=\pi(\tilde{b})$.

A set of the form $B=L \cap S_{\tilde{b}}$ where $\tilde{b} \in \operatorname{int}\left(B(\mathcal{H})^{+}\right)$and $L \subseteq B_{h}(\mathcal{H})$ is a subspace will be called a section of a base of $B(\mathcal{H})^{+}$, or simply a section. Let $J=\operatorname{span}(B)$ be the real linear span of $B$, then

$$
B \subseteq J \cap S_{\tilde{b}} \subseteq L \cap S_{\tilde{b}}=B,
$$

so that $B=J \cap S_{\tilde{b}}$ and $B$ is a base of $Q=J \cap B(\mathcal{H})^{+}$. If moreover $B$ contains a positive definite element, we say that $B$ is a faithful section. In this case, we have $B \cap \operatorname{int}\left(B(\mathcal{H})^{+}\right)=\operatorname{ri}(B)$, where $\operatorname{ri}(B)$ denotes the relative interior of $B$. Indeed, since $B=L_{\tilde{b}} \cap B(\mathcal{H})^{+}$, where $L_{\tilde{b}}=: L \cap \mathcal{T}_{\tilde{b}}$ is an affine subspace and $L_{\tilde{b}} \cap \operatorname{int}\left(B(\mathcal{H})^{+}\right) \neq \emptyset$, we have by [19, Theorem 6.5] that

$$
\operatorname{ri}(B)=\operatorname{ri}\left(L_{\tilde{b}}\right) \cap \operatorname{ri}\left(B(\mathcal{H})^{+}\right)=L_{\tilde{b}} \cap \operatorname{int}\left(B(\mathcal{H})^{+}\right)=B \cap \operatorname{int}\left(B(\mathcal{H})^{+}\right)
$$

For example, note that if $B=\{b\}$ for some $b \in B(\mathcal{H})^{+}$, then $B$ is a section and $B$ is faithful if and only if $b$ is positive definite. If a section $B$ is not faithful, then there is some element $b \in B$ such that $p=\operatorname{supp}(b)$ and $B \subset B(p \mathcal{H})$. Then $B$ is a faithful section of a base of $B(p \mathcal{H})^{+}$, in this case, $r i(B)=B \cap \operatorname{ri}\left(B(p \mathcal{H})^{+}\right)$. From now on, we will suppose that $B$ is a faithful section and we put $J:=\operatorname{span}(B)$, $Q:=J \cap B(\mathcal{H})^{+}$.

Note that in Lemma 2, the correspondence between the base $B$ and the element $\tilde{b}$ such that $B=J \cap S_{\tilde{b}}$ is not one-to-one, since the order unit $e_{B}=\pi(\tilde{b})$ may contain more different positive definite elements. We will now look at the set of all such elements. Let

$$
\tilde{B}:=\left\{\tilde{b} \in B(\mathcal{H})^{+}, \operatorname{Tr} b \tilde{b}=1, b \in B\right\}
$$


Note that

$$
\tilde{B}=\pi^{-1}\left(e_{B}\right) \cap B(\mathcal{H})^{+}=\left(\tilde{b}+J^{\perp}\right) \cap B(\mathcal{H})^{+},
$$

where $\tilde{b}$ is any element in $\tilde{B}$. Let $\tilde{J}:=\operatorname{span}(\tilde{B})$ and $\tilde{Q}:=\tilde{J} \cap B(\mathcal{H})^{+}$. Note that $\tilde{B}$ always contains a positive definite element, so that $\tilde{J}$ is positively generated and $\tilde{Q}$ is a proper cone in $\tilde{J}$. Since by (8) $\tilde{B}$ is an intersection of $B(\mathcal{H})^{+}$by an affine subspace, we have

$$
\left\{\tilde{b} \in \operatorname{int}\left(B(\mathcal{H})^{+}\right), B=J \cap S_{\tilde{b}}\right\}=\tilde{B} \cap \operatorname{int}\left(B(\mathcal{H})^{+}\right)=\operatorname{ri}(\tilde{B}) .
$$

Lemma 3. Let $b \in \operatorname{ri}(B), \tilde{b} \in \operatorname{ri}(\tilde{B})$. Then

(i) $\tilde{B}=\tilde{J} \cap S_{b}$, so that $\tilde{B}$ is a faithful section of a base of $B(\mathcal{H})^{+}$.

(ii) $\tilde{\tilde{B}}=B$.

(iii) $B=\bigcap_{\tilde{b}^{\prime} \in \operatorname{ri}(\tilde{B})} S_{\tilde{b}^{\prime}}$.

Proof. (i) Since $\tilde{B}$ is convex, any element $y \in \tilde{J}$ has the form $y=\lambda \tilde{b}_{1}-\mu \tilde{b}_{2}$, with $\tilde{b}_{1}, \tilde{b}_{2} \in \tilde{B}$ and $\lambda, \mu \geq 0$. Hence by (8), $y=(\lambda-\mu) \tilde{b}+z$ for some $z \in J^{\perp}$. If $y \in \tilde{J} \cap S_{b}$, we must have $1=\operatorname{Tr} y b=\lambda-\mu$, so that $y \in\left(\tilde{b}+J^{\perp}\right) \cap B(\mathcal{H})^{+}=\tilde{B}$. The opposite inclusion is obvious.

(ii) It is clear that $B \subset \underset{\tilde{B}}{\tilde{J}}$ and $\tilde{\tilde{B}}$ is a base of $\tilde{\tilde{Q}}=\tilde{\tilde{J}} \cap B(\mathcal{H})^{+}$. Consequently, it is enough to prove that $\tilde{\tilde{J}}=J$, since then $B$ and $\tilde{\tilde{B}}$ are two bases of the same cone. By the proof of (i), $\tilde{J}=\operatorname{span}\{\tilde{b}\} \vee J^{\perp}$ and similarly,

$$
\tilde{\tilde{J}}=\operatorname{span}\{b\} \vee \tilde{J}^{\perp}=\operatorname{span}\{b\} \vee\left(\{\tilde{b}\}^{\perp} \wedge J\right)=\left(\operatorname{span}\{b\} \vee\{\tilde{b}\}^{\perp}\right) \wedge J=J,
$$

here the third equality follows from $b \in J$ and the last equality follows from the fact that $\{\tilde{b}\}^{\perp}$ is a subspace of codimension 1 not containing $b$, so that $\operatorname{span}\{b\} \vee\{\tilde{b}\}^{\perp}=B_{h}(\mathcal{H})$.

(iii) It is clear that $B \subseteq \bigcap_{\tilde{b}^{\prime} \in \operatorname{ri}(\tilde{B})} S_{\tilde{b}^{\prime}}$. If $a \in \bigcap_{\tilde{b}^{\prime} \in \operatorname{ri}(\tilde{B})} S_{\tilde{b}^{\prime}}$, then $a$ is a positive element such that $\operatorname{Tr} a \tilde{b}^{\prime}=1$ for all $\tilde{b}^{\prime} \in \operatorname{cl}(\operatorname{ri}(\tilde{B}))=\tilde{B}$, hence $a \in \tilde{\tilde{B}}=B$.

We call $\tilde{B}$ the dual section of $B$. Since $B$ is a base of $Q$, the base norm $\|\cdot\|_{B}$ is defined in $J$. Next we show that this norm can be naturally extended to $B_{h}(\mathcal{H})$. For this, let us define

$$
\mathcal{O}_{B}:=\left\{x \in B_{h}(\mathcal{H}), x=x_{1}-x_{2}, x_{1}, x_{2} \in B(\mathcal{H})^{+}, x_{1}+x_{2} \in B\right\}
$$

For $b \in B(\mathcal{H})^{+}$, we define $\mathcal{O}_{b}:=\mathcal{O}_{\{b\}}$.

Lemma 4. We have

(i) $\mathcal{O}_{B}=\left\{x \in B_{h}(\mathcal{H}), \exists b^{\prime} \in B,-b^{\prime} \leq x \leq b^{\prime}\right\}=\bigcup_{b^{\prime} \in B} \mathcal{O}_{b^{\prime}}$

(ii) The unit ball of the base norm $\|\cdot\|_{B}$ in $J$ is $\mathcal{O}_{B} \cap J$. 
Proof. (i) Let $x=x_{1}-x_{2}$ with $x_{1}+x_{2}=b^{\prime} \in B$, then $-b^{\prime}=-\left(x_{1}+x_{2}\right) \leq x \leq$ $x_{1}+x_{2}=b^{\prime}$. Conversely, let $-b^{\prime} \leq x \leq b^{\prime}$ for some $b^{\prime} \in B$. Put $x_{ \pm}=1 / 2\left(b^{\prime} \pm x\right)$, then $x_{ \pm} \in B(\mathcal{H})^{+}, x_{+}-x_{-}=x$ and $x_{+}+x_{-}=b^{\prime} \in B$.

(ii) By definiton, the unit ball of $\|\cdot\|_{B}$ in $J$ is the set of elements of $J$ of the form $x=\lambda b_{1}-(1-\lambda) b_{2}, b_{1}, b_{2} \in B, 0 \leq \lambda \leq 1$. Then clearly $x \in \mathcal{O}_{B}$, by putting $x_{1}=\lambda b_{1}$ and $x_{2}=(1-\lambda) b_{2}$. Conversely, let $x \in J$ be such that $-b^{\prime} \leq x \leq b^{\prime}$ for some $b^{\prime} \in B$, and put $x_{ \pm}=1 / 2\left(b^{\prime} \pm x\right)$, then $x_{ \pm} \in B(\mathcal{H})^{+} \cap J=Q$. Let $x_{ \pm}=\lambda_{ \pm} b_{ \pm}$, for $\lambda_{ \pm} \geq 0, b_{ \pm} \in B$, then by applying the corresponding order unit $e_{B}$ to the equality $b^{\prime}=x_{+}+x_{-}$, we see that we must have $\lambda_{+}+\lambda_{-}=1$, so that $\|x\|_{B} \leq 1$.

Theorem 1. Let $B$ be a faithful section and let $\tilde{B}$ be the dual section. Then $\mathcal{O}_{B}$ is the unit ball of a norm in $B_{h}(\mathcal{H})$. The unit ball of the dual norm is $\mathcal{O}_{\tilde{B}}$.

We will denote this norm by $\|\cdot\|_{B}$, note that Lemma 4 (ii) justifies this notation.

Proof. It is clear that $\mathcal{O}_{B}$ is convex and symmetric, that is, $-\mathcal{O}_{B} \subseteq \mathcal{O}_{B}$. Since $B$ is compact, $\mathcal{O}_{B}$ is closed. If $x \in \mathcal{O}_{B}$, then $x=x_{1}-x_{2}$ with $x_{1}, x_{2} \geq 0$, $x_{1}+x_{2} \in B$ and by (6),

$$
\|x\|_{S_{\tilde{b}}} \leq\left\|x_{1}\right\|_{S_{\tilde{b}}}+\left\|x_{2}\right\|_{S_{\tilde{b}}}=\operatorname{Tr}\left(x_{1}+x_{2}\right) \tilde{b}=1
$$

for any $\tilde{b} \in \operatorname{ri}(\tilde{B})$, hence $\mathcal{O}_{B}$ is bounded. Moreover, since $b \in \operatorname{ri}(B)$ is an order unit, for every $x \in B_{h}(\mathcal{H})$ there is some $t>0$ such that $-t b \leq x \leq t b$, so that $\mathcal{O}_{B}$ is absorbing (see Lemma $4(\mathrm{i})$ ). These facts imply that $\mathcal{O}_{B}$ is the unit ball of a norm.

To show duality of the norms $\|\cdot\|_{B}$ and $\|\cdot\|_{\tilde{B}}$, let $\mathcal{H}_{2}=\mathcal{H} \oplus \mathcal{H}$ and let $\Phi: B_{h}\left(\mathcal{H}_{2}\right) \rightarrow B_{h}(\mathcal{H})$ be the map defined by $\Phi(a \oplus b)=a+b$. Let $J_{2}=\Phi^{-1}(J)$, then $J_{2}$ is a subspace in $B_{h}\left(\mathcal{H}_{2}\right)$ and

$$
J_{2}^{\perp}=\Phi^{*}\left(J^{\perp}\right)=\left\{x \oplus x, x \in J^{\perp}\right\},
$$

see [13]. Let $\pi_{2}: B\left(\mathcal{H}_{2}\right) \rightarrow J_{2}^{*}=\left.B\left(\mathcal{H}_{2}\right)\right|_{J_{2}^{\perp}}$ be the quotient map.

Let $\tilde{b} \in \operatorname{ri}(\tilde{B})$ and put $B_{2}=J_{2} \cap S_{\tilde{b} \oplus \tilde{b}}$. Then $B_{2}$ is a base of $Q_{2}=J_{2} \cap B\left(\mathcal{H}_{2}\right)^{+}$ and it is clear that for $w_{1}, w_{2} \in B(\mathcal{H})^{+}, w_{1} \oplus w_{2} \in B_{2}$ if and only if $w_{1}+w_{2} \in B$. Let now $a \in B_{h}(\mathcal{H})$, then $a \in \mathcal{O}_{B}^{\circ}$ if and only if $\operatorname{Tr}(a \oplus-a) w \leq 1$ for all $w \in B_{2}$. Equivalently,

$$
\pi_{2}(a \oplus-a) \leq_{Q_{2}^{*}} e_{B_{2}}=\pi_{2}(\tilde{b} \oplus \tilde{b}),
$$

that is, there is some $v \in J_{2}^{\perp}$ such that $a \oplus-a \leq \tilde{b} \oplus \tilde{b}+v$. Since $v=x \oplus x$, $x \in J^{\perp}$, we obtain $\pm a \leq \tilde{b}+x$. Note that we must have $\tilde{b}+x \geq 0$ : if $c$ is any element in $B(\mathcal{H})^{+}$, then we have $\pm \operatorname{Tr} c a \leq \operatorname{Tr} c(b+x)$, so that $\operatorname{Tr} c(b+x)$ cannot be negative. Hence $\pm a \leq \tilde{b}+x \in \tilde{B}$, so that $a \in \mathcal{O}_{\tilde{B}}$, by Lemma 4 (i).

Corollary 2. Let $x \in B_{h}(\mathcal{H})$. Then 
(i) $\mathcal{O}_{B}=\bigcap_{\tilde{b} \in \operatorname{ri}(\tilde{B})} \mathcal{O}_{S_{\tilde{b}}}$,

(ii) $\|x\|_{B}=\sup _{\tilde{b} \in \operatorname{ri(\tilde {B})}}\|x\|_{S_{\tilde{b}}}=\sup _{\tilde{b} \in \tilde{B}}\left\|\tilde{b}^{1 / 2} x \tilde{b}^{1 / 2}\right\|_{1}$

(iii) $\|x\|_{B}=\inf _{b \in r i(B)}\|x\|_{b}=\inf _{b \in B}\left\|b^{-1 / 2} x b^{-1 / 2}\right\|$.

Proof. (i) It is easy to see from Lemma 4 that

$$
\mathcal{O}_{B}=\bigcup_{b \in B} \mathcal{O}_{b}=\operatorname{cl}\left(\bigcup_{b \in \operatorname{ri}(B)} \mathcal{O}_{b}\right) .
$$

Indeed, let $x \in B_{h}(\mathcal{H})$ be such that $-b \leq x \leq b$ for some $b \in B$ and let $b^{\prime} \in \operatorname{ri}(B)$, then $b_{\epsilon}:=\epsilon b^{\prime}+(1-\epsilon) b \in \operatorname{ri}(B)$ for all $0<\epsilon<1$. Let $x^{\prime} \in \mathcal{O}_{b^{\prime}}$ be any element, then $x_{\epsilon}:=\epsilon x^{\prime}+(1-\epsilon) x \in \mathcal{O}_{b_{\epsilon}}$ and $x=\lim _{\epsilon \rightarrow 0^{+}} x_{\epsilon} \in \operatorname{cl}\left(\bigcup_{b \in \operatorname{ri}(B)} \mathcal{O}_{b}\right)$.

Since $A^{\circ}=(c l(\operatorname{conv}(A)))^{\circ}$ for any subset $A \in B_{h}(\mathcal{H})$ containing 0 , we obtain by Theorem 1 that

$$
\mathcal{O}_{B}=\mathcal{O}_{\tilde{B}}^{\circ}=\left(\bigcup_{\tilde{b} \in \operatorname{ri}(\tilde{B})} \mathcal{O}_{\tilde{b}}\right)^{\circ}=\bigcap_{\tilde{b} \in \operatorname{ri}(\tilde{B})} \mathcal{O}_{\tilde{b}}^{\circ}=\bigcap_{\tilde{b} \in \operatorname{ri}(\tilde{B})} \mathcal{O}_{S_{\tilde{b}}}
$$

(ii) Since $\mathcal{O}_{B}$ is the unit ball of $\|\cdot\|_{B}$, we get from (i)

$$
\begin{aligned}
\|x\|_{B} & =\inf \left\{\lambda>0, x \in \lambda \mathcal{O}_{B}\right\}=\inf \left\{\lambda>0, x \in \lambda \mathcal{O}_{S_{\tilde{b}}}, \forall \tilde{b} \in \operatorname{ri}(\tilde{B})\right\} \\
& =\inf \left\{\lambda>0, \lambda \geq\|x\|_{S_{\tilde{b}}}, \forall \tilde{b} \in \operatorname{ri}(\tilde{B})\right\}=\sup _{\tilde{b} \in \operatorname{ri}(\tilde{B})}\|x\|_{S_{\tilde{b}}}=\sup _{\tilde{b} \in \tilde{B}}\left\|\tilde{b}^{1 / 2} x \tilde{b}^{1 / 2}\right\|_{1},
\end{aligned}
$$

the last equality follows from (6) and continuity of the norm $\|\cdot\|_{1}$.

(iii) On the other hand, we get from Lemma 4 and (10)

$$
\begin{aligned}
\|x\|_{B} & =\inf \left\{\lambda>0, x \in \lambda \mathcal{O}_{B}\right\}=\inf \left\{\lambda>0, x \in \lambda \cup_{b \in r i(B)} \mathcal{O}_{b}\right\} \\
& =\inf _{b \in r i(B)} \inf \left\{\lambda>0, x \in \lambda \mathcal{O}_{b}\right\}=\inf _{b \in r i(B)}\|x\|_{b}=\inf _{b \in B}\left\|b^{-1 / 2} x b^{-1 / 2}\right\|
\end{aligned}
$$

where the last equality follows by (7).

Corollary 3. For $a \in B(\mathcal{H})^{+}$, we have

$$
\|a\|_{B}=\sup _{\tilde{b} \in \tilde{B}} \operatorname{Tr} a \tilde{b}=\inf _{b \in B} 2^{D_{\max }(a \| b)}
$$

Proof. We have

$$
\|a\|_{B}=\sup _{x \in \mathcal{O}_{\tilde{B}}} \operatorname{Tr} a x
$$

Let $x \in \mathcal{O}_{\tilde{B}}$, then $x=x_{1}-x_{2}, x_{1}, x_{2} \in B(\mathcal{H})^{+}$and $x_{1}+x_{2}=: \tilde{b}_{x} \in \tilde{B}$, so that

$$
\operatorname{Tr} a x \leq \operatorname{Tr} a x_{1} \leq \operatorname{Tr} a \tilde{b}_{x} \leq \sup _{\tilde{b} \in \tilde{B}} \operatorname{Tr} a \tilde{b} \leq \sup _{y \in \mathcal{O}_{\tilde{B}}} \operatorname{Tr} a y=\|a\|_{B}
$$

Hence $\|a\|_{B}=\sup _{\tilde{b} \in \tilde{B}} \operatorname{Tr} a \tilde{b}$. The second equality follows directly from Corollary 2 (iii) and the definition of $D_{\max }$. 
We can also characterize the maximizer resp. minimizer in Corollary 3 ,

Corollary 4. Let $a \in B(\mathcal{H})^{+}$.

(i) Let $\tilde{b}_{0} \in \tilde{B}$, then $\|a\|_{B}=\operatorname{Tr} a \tilde{b}_{0}$ if and only if there exists some $q \in Q$, such that $a \leq q$ and $(q-a) \tilde{b}_{0}=0$. In this case, $q=\|a\|_{B} b_{0}$ and $\|a\|_{B}=$ $2^{D_{\max }\left(a \| b_{0}\right)}$.

(ii) Let $b_{0} \in B$, then $\|a\|_{B}=2^{D_{\max }\left(a \| b_{0}\right)}$ if and only if there exists some $t>0$ and $\tilde{b}_{0} \in \tilde{B}$, such that $a \leq t b_{0}$ and $\left(t b_{0}-a\right) \tilde{b}_{0}=0$. In this case, $t=\|a\|_{B}=\operatorname{Tr} a \tilde{b}_{0}$.

Proof. (i) Let $\tilde{b}_{0} \in \tilde{B}$ be such that $\|a\|_{B}=\operatorname{Tr} a \tilde{b}_{0}$. Let $b_{0} \in B$ be such that $\|a\|_{B}=2^{D_{\max }\left(a \| b_{0}\right)}$, in particular, $a \leq\|a\|_{B} b_{0}$. Put $q=\|a\|_{B} b_{0}$, then $q-a \geq 0$ and $\operatorname{Tr}(q-a) \tilde{b}_{0}=0$. Since also $\tilde{b}_{0} \geq 0$, it follows that $(q-a) \tilde{b}_{0}=0$.

Conversely, suppose $q \in Q$ satisfies $a \leq q$ and $(q-a) \tilde{b}_{0}=0$. Then $q=s b_{0}$ for some $b_{0} \in B, s \geq 0$. Since $a \leq s b_{0}$, we have

$$
\|a\|_{B} \leq s=\operatorname{Tr} a \tilde{b}_{0} \leq\|a\|_{B},
$$

so that $\operatorname{Tr} a \tilde{b}_{0}=\|a\|_{B}=s=2^{D_{\max }\left(a \| b_{0}\right)}$.

(ii) is proved similarly.

\section{Generalized channels}

Let $B$ be a section of a base of $B(\mathcal{H})^{+}$. A generalized channel with respect to $B$ (or a $B$-channel) is a completely positive map $\Phi: B(\mathcal{H}) \rightarrow B(\mathcal{K})$ such that $\Phi(B) \subseteq \mathfrak{S}(\mathcal{K})$. Let $X_{\Phi}$ be the Choi matrix of $\Phi$, then $\Phi$ is a generalized channel with respect to $B$ if and only if $X_{\Phi} \geq 0$ and

$$
1=\operatorname{Tr} \Phi(b)=\operatorname{Tr} \operatorname{Tr} \mathcal{H}\left[\left(I \otimes b^{\top}\right) X_{\Phi}\right]=\operatorname{Tr}\left(I \otimes b^{\top}\right) X_{\Phi}=\operatorname{Tr} b^{\top} \operatorname{Tr}_{\mathcal{K}} X_{\Phi}
$$

for all $b \in B$. Let $\mathcal{C}_{B}(\mathcal{H}, \mathcal{K})$ denote the set of Choi matrices of all generalized channels with respect to $B$, then

$$
\mathcal{C}_{B}(\mathcal{H}, \mathcal{K})=\left\{X \in B(\mathcal{K} \otimes \mathcal{H})^{+}, \operatorname{Tr}_{\mathcal{K}} X \in \tilde{B}^{\top}\right\} .
$$

Let us remark that if $B$ is a section, then $B^{\top}:=\left\{b^{\top}, b \in B\right\}$ is a section as well, here $b^{\top}$ denotes the transpose of $b$. Moreover, $\widetilde{B^{\top}}=\tilde{B}^{\top}$. Note also that we have

$$
\mathcal{C}_{B}(\mathcal{H}, \mathbb{C})=\tilde{B}^{\top}
$$

so that, in particular, $\mathcal{C}_{B}(\mathcal{H}, \mathbb{C})$ is a section.

Proposition 1. Let $B$ be a faithful section of a base of $B(\mathcal{H})^{+}$. Then $\mathcal{C}_{B}(\mathcal{H}, \mathcal{K})$ is a faithful section of a base of $B(\mathcal{K} \otimes \mathcal{H})^{+}$and $\widehat{\mathcal{C}_{B}(\mathcal{H}, \mathcal{K})}=\left\{I_{\mathcal{K}} \otimes b^{\top}, b \in B\right\}$. 
Proof. It is easy to see that $I_{\mathcal{K}} \otimes B^{\top}=\left\{I_{\mathcal{K}} \otimes b^{\top}, b \in B\right\}$ is a faithful section of a base of $B(\mathcal{K} \otimes \mathcal{H})^{+}$and

$$
\mathcal{C}_{B}(\mathcal{H}, \mathcal{K})=\left\{X \in B(\mathcal{K} \otimes \mathcal{H})^{+}, \operatorname{Tr} X\left(I \otimes b^{\top}\right)=1, \forall b \in B\right\}=\widetilde{I_{\mathcal{K}} \otimes B^{\top}} .
$$

The proof now follows by Lemma 3 (i) and (ii).

Let now $X \in B_{h}(\mathcal{K} \otimes \mathcal{H})$ and let $\Phi: B(\mathcal{H}) \rightarrow B(\mathcal{K})$ be the corresponding Hermitian map. By Corollary 2 and Proposition 1 ,

$$
\|X\|_{\mathcal{C}_{B}(\mathcal{H}, \mathcal{K})}=\sup _{b \in B}\left\|\left(I \otimes\left(b^{\top}\right)^{1 / 2}\right) X\left(I \otimes\left(b^{\top}\right)^{1 / 2}\right)\right\|_{1}
$$

and we have

$$
\left(I \otimes\left(b^{\top}\right)^{1 / 2}\right) X\left(I \otimes\left(b^{\top}\right)^{1 / 2}\right)=\left(\Phi \otimes i d_{\mathcal{H}}\right)\left(\sigma_{b}\right),
$$

where $\sigma_{b}=\left|\psi_{b}\right\rangle\left\langle\psi_{b}\right|$, with

$$
\left|\psi_{b}\right\rangle=\sum_{i}|i\rangle \otimes\left(b^{\top}\right)^{1 / 2}|i\rangle=\sum_{i} b^{1 / 2}|i\rangle \otimes|i\rangle \in \mathcal{H} \otimes \mathcal{H}
$$

Hence $\sigma_{b} \in B(\mathcal{H} \otimes \mathcal{H})^{+}$and $\operatorname{Tr}_{1} \sigma_{b}=b^{\top} \in B^{\top}$, so that $\sigma_{b} \in \mathcal{C}_{\tilde{B}}(\mathcal{H}, \mathcal{H})$. Conversely, if $\sigma=|\varphi\rangle\langle\varphi| \in \mathcal{C}_{\tilde{B}}(\mathcal{H}, \mathcal{L})$ for some Hilbert space $\mathcal{L}$, then there is some linear map $R: \mathcal{H} \rightarrow \mathcal{L}$ satisfying $R^{*} R=b \in B$ and such that $|\varphi\rangle=\sum_{i} R|i\rangle \otimes|i\rangle$. Let $U: \mathcal{H} \rightarrow \mathcal{L}$ be an isometry such that $R=U b^{1 / 2}$, then

$$
|\varphi\rangle=\sum_{i} R|i\rangle \otimes|i\rangle=\sum_{i} U b^{1 / 2}|i\rangle \otimes|i\rangle=(U \otimes I)\left|\psi_{b}\right\rangle
$$

Theorem 2. Let $X \in B_{h}(\mathcal{K} \otimes \mathcal{H})$ and let $\Phi$ be the corresponding Hermitian map $B(\mathcal{H}) \rightarrow B(\mathcal{K})$. Let $\mathcal{L}$ be any Hilbert space with $\operatorname{dim}(\mathcal{L})=\operatorname{dim}(\mathcal{H})$. Then

$$
\begin{aligned}
\|X\|_{\mathcal{C}_{B}(\mathcal{H}, \mathcal{K})} & =\sup _{\operatorname{dim}\left(\mathcal{L}^{\prime}\right)<\infty} \sup _{\sigma \in \mathcal{C}_{\tilde{B}}\left(\mathcal{H}, \mathcal{L}^{\prime}\right)}\left\|\left(\Phi \otimes i d_{\mathcal{L}^{\prime}}\right)(\sigma)\right\|_{1} \\
& =\sup _{\sigma \in \mathcal{C}_{\tilde{B}}(\mathcal{H}, \mathcal{L})}\left\|\left(\Phi \otimes i d_{\mathcal{L}}\right)(\sigma)\right\|_{1}
\end{aligned}
$$

and the dual norm is $\|X\|_{\mathcal{C}_{B}(\mathcal{H}, \mathcal{K})}^{*}=\|X\|_{I_{\mathcal{K}} \otimes B^{\top}}$. Moreover, if $X \geq 0$ then

$$
\|X\|_{\mathcal{C}_{B}(\mathcal{H}, \mathcal{K})}=\sup _{b \in B} \operatorname{Tr} \Phi(b)=\inf _{Y \in \mathcal{C}_{B}(\mathcal{H}, \mathcal{K})} 2^{D_{\max }(X \| Y)}
$$

and

$$
\|X\|_{I \otimes B^{\top}}=\inf _{b \in B} 2^{D_{\max }\left(X \| I \otimes b^{\top}\right)}=\sup _{Y \in \mathcal{C}_{B}(\mathcal{H}, \mathcal{K})} \operatorname{Tr} X Y=\sup _{S}\left\langle\psi\left|X_{S^{*} \circ \Phi}\right| \psi\right\rangle,
$$

where the last supremum is taken over the set of all $B$-channels $B(\mathcal{H}) \rightarrow B(\mathcal{K})$. 
Proof. From what was said above, it is easy to see that

$$
\|X\|_{\mathcal{C}_{B}(\mathcal{H}, \mathcal{K})}=\sup _{|\varphi\rangle\langle\varphi| \in \mathcal{C}_{\tilde{B}}(\mathcal{H}, \mathcal{L})}\left\|\left(\Phi \otimes i d_{\mathcal{L}}\right)(|\varphi\rangle\langle\varphi|)\right\|_{1}
$$

with $\operatorname{dim}(\mathcal{L})=\operatorname{dim}(\mathcal{H})$. We will show that

$$
\sup _{|\varphi\rangle\langle\varphi| \in \mathcal{C}_{\tilde{B}}\left(\mathcal{H}, \mathcal{L}^{\prime}\right)}\left\|\left(\Phi \otimes i d_{\mathcal{L}^{\prime}}\right)(|\varphi\rangle\langle\varphi|)\right\|_{1} \leq \sup _{|\varphi\rangle\langle\varphi| \in \mathcal{C}_{\tilde{B}}(\mathcal{H}, \mathcal{L})}\left\|\left(\Phi \otimes i d_{\mathcal{L}}\right)(|\varphi\rangle\langle\varphi|)\right\|_{1}
$$

whenever $\operatorname{dim}\left(\mathcal{L}^{\prime}\right) \geq \operatorname{dim}(\mathcal{L})$. The proof is almost the same as the proof of [20, Theorem 5], we include it here for completeness.

So let $\operatorname{dim}\left(\mathcal{L}^{\prime}\right) \geq \operatorname{dim}(\mathcal{L})=\operatorname{dim}(\mathcal{H})$, then there is some $\varphi_{0} \in \mathcal{H} \otimes \mathcal{L}^{\prime}$, with $\left|\varphi_{0}\right\rangle\left\langle\varphi_{0}\right| \in \mathcal{C}_{\tilde{B}}\left(\mathcal{H}, \mathcal{L}^{\prime}\right)$ such that

$$
\sup _{|\varphi\rangle\langle\varphi| \in \mathcal{C}_{\tilde{B}}\left(\mathcal{H}, \mathcal{L}^{\prime}\right)}\left\|\Phi \otimes i d_{\mathcal{L}^{\prime}}(|\varphi\rangle\langle\varphi|)\right\|_{1}=\left\|\Phi \otimes i d_{\mathcal{L}^{\prime}}\left(\left|\varphi_{0}\right\rangle\left\langle\varphi_{0}\right|\right)\right\|_{1}
$$

Let $\left|\varphi_{0}\right\rangle=\sum_{i=1}^{m} s_{i}\left|\varphi_{i}\right\rangle \otimes\left|\xi_{i}\right\rangle$ be the Schmidt decomposition of $\varphi_{0}$, with $\left\{\left|\varphi_{i}\right\rangle\right\}$ and $\left\{\left|\xi_{i}\right\rangle\right\}$ orthonormal sets in $\mathcal{H}$ resp. $\mathcal{L}^{\prime}$ and $m=\operatorname{dim}(\mathcal{H})$. Then $\left|\varphi_{0}\right\rangle\left\langle\varphi_{0}\right|=$ $\sum_{i, j}\left|\varphi_{i}\right\rangle\left\langle\varphi_{j}|\otimes| \xi_{i}\right\rangle\left\langle\xi_{j}\right|$ and

$$
\left(\operatorname{Tr}_{\mathcal{L}^{\prime}}\left|\varphi_{0}\right\rangle\left\langle\varphi_{0}\right|\right)^{\top}=\left(\sum_{i} s_{i}\left|\varphi_{i}\right\rangle\left\langle\varphi_{i}\right|\right)^{\top} \in \tilde{\tilde{B}}=B
$$

Let $\left\{\left|e_{i}\right\rangle, i=1, \ldots, m\right\}$ be an ONB in $\mathcal{L}$. Define the linear map $U: \mathcal{L}^{\prime} \rightarrow \mathcal{L}$ by $U=\sum_{i=1}^{m}\left|e_{i}\right\rangle\left\langle\xi_{i}\right|$, then $U^{*} U=\sum_{i}\left|\xi_{i}\right\rangle\left\langle\xi_{i}\right|$ is the projection in $\mathcal{L}^{\prime}$ onto the subspace spanned by the vectors $\left|\xi_{i}\right\rangle, i=1, \ldots, m$ and $\left(I \otimes U^{*} U\right)\left|\varphi_{0}\right\rangle=\left|\varphi_{0}\right\rangle$. Put $\varphi_{U}:=(I \otimes U)\left|\varphi_{0}\right\rangle=\sum_{i}\left|\varphi_{i}\right\rangle \otimes\left|e_{i}\right\rangle$, then it is easy to see that $\left|\varphi_{U}\right\rangle\left\langle\varphi_{U}\right| \in$ $\mathcal{C}_{\tilde{B}}(\mathcal{H}, \mathcal{L})$. Now we have

$$
\begin{aligned}
\sup _{|\varphi\rangle\langle\varphi| \in \mathcal{C}_{\tilde{B}}(\mathcal{H}, \mathcal{L})}\left\|\Phi \otimes i d_{\mathcal{L}}(|\varphi\rangle\langle\varphi|)\right\|_{1} & \geq\left\|\Phi \otimes i d_{\mathcal{L}}\left(\left|\varphi_{U}\right\rangle\left\langle\varphi_{U}\right|\right)\right\|_{1} \\
& \geq\left\|\left(I \otimes U^{*}\right)\left(\Phi \otimes i d_{\mathcal{L}}\right)\left(\left|\varphi_{U}\right\rangle\left\langle\varphi_{U}\right|\right)(I \otimes U)\right\|_{1} \\
& =\left\|\Phi \otimes i d_{\mathcal{L}^{\prime}}\left(\left(I \otimes U^{*}\right)\left|\varphi_{U}\right\rangle\left\langle\varphi_{U}\right|(I \otimes U)\right)\right\|_{1} \\
& =\left\|\Phi \otimes i d_{\mathcal{L}^{\prime}}\left(\left|\varphi_{0}\right\rangle\left\langle\varphi_{0}\right|\right)\right\|_{1} \\
& =\sup _{|\varphi\rangle\langle\varphi| \in \mathcal{C}_{\tilde{B}}\left(\mathcal{H}, \mathcal{L}^{\prime}\right)}\left\|\Phi \otimes i d_{\mathcal{L}^{\prime}}(|\varphi\rangle\langle\varphi|)\right\|_{1}
\end{aligned}
$$

Next, let $Y$ be any element in $\mathcal{C}_{\tilde{B}}\left(\mathcal{H}, \mathcal{L}^{\prime}\right)$, then the corresponding map $\xi$ : $B(\mathcal{H}) \rightarrow B\left(\mathcal{L}^{\prime}\right)$ has the form

$$
\xi(a)=\sum_{i=1}^{N} V_{i} a V_{i}^{*}, \quad a \in B(\mathcal{H})
$$

where $V_{i}: \mathcal{H} \rightarrow \mathcal{L}^{\prime}$ are linear maps such that $\sum_{i} V_{i}^{*} V_{i} \in B$. Let $\mathcal{L}_{0}^{\prime}$ be a Hilbert space with $\operatorname{dim}\left(\mathcal{L}_{0}^{\prime}\right)=N$ and let $\left\{\left|f_{j}\right\rangle, j=1, \ldots, N\right\}$ be an ONB in $\mathcal{L}_{0}^{\prime}$. Define $V=\sum_{j=1}^{N} V_{j} \otimes\left|f_{j}\right\rangle$, then $V$ is a linear map $\mathcal{H} \rightarrow \mathcal{L}^{\prime} \otimes \mathcal{L}_{0}^{\prime}$ with 
$V^{*} V=\sum_{i} V_{i}^{*} V_{i} \in B$. Let $\mathcal{V}(a)=V a V^{*}$ and let $Z$ be the Choi matrix of $\mathcal{V}$, then $Z$ is a rank one element in $\mathcal{C}_{\tilde{B}}\left(\mathcal{H}, \mathcal{L}^{\prime} \otimes \mathcal{L}_{0}^{\prime}\right)$. Moreover, $\xi(a)=\operatorname{Tr}_{\mathcal{L}_{0}^{\prime}} V a V^{*}$ and $Y=\operatorname{Tr}_{\mathcal{L}_{0}^{\prime}} Z$. It follows that

$$
\begin{aligned}
\left\|\left(\Phi \otimes i d_{\mathcal{L}^{\prime}}\right)(Y)\right\|_{1} & =\left\|\left(\Phi \otimes i d_{\mathcal{L}^{\prime}}\right)\left(\operatorname{Tr}_{\mathcal{L}_{0}^{\prime}} Z\right)\right\|_{1}=\left\|\operatorname{Tr}_{\mathcal{L}_{0}^{\prime}}\left(\Phi \otimes i d_{\mathcal{L}^{\prime} \otimes \mathcal{L}_{0}^{\prime}}\right)(Z)\right\|_{1} \\
& \leq\left\|\left(\Phi \otimes i d_{\mathcal{L}^{\prime} \otimes \mathcal{L}_{0}^{\prime}}\right)(Z)\right\|_{1} \leq\|X\|_{\mathcal{C}_{B}(\mathcal{H}, \mathcal{K})}
\end{aligned}
$$

We now have

$$
\begin{aligned}
\|X\|_{\mathcal{C}_{B}(\mathcal{H}, \mathcal{K})} & =\sup _{|\varphi\rangle\langle\varphi| \in \mathcal{C}_{\tilde{B}}(\mathcal{H}, \mathcal{L})}\left\|\left(\Phi \otimes i d_{\mathcal{L}}\right)(|\varphi\rangle\langle\varphi|)\right\|_{1} \leq \sup _{\sigma \in \mathcal{C}_{\tilde{B}}(\mathcal{H}, \mathcal{L})}\left\|\left(\Phi \otimes i d_{\mathcal{L}}\right)(\sigma)\right\|_{1} \\
& \leq \sup _{\operatorname{dim}\left(\mathcal{L}^{\prime}\right)<\infty} \sup _{\sigma \in \mathcal{C}_{\tilde{B}}\left(\mathcal{H}, \mathcal{L}^{\prime}\right)}\left\|\left(\Phi \otimes i d_{\mathcal{L}^{\prime}}\right)(\sigma)\right\|_{1} \leq\|X\|_{\mathcal{C}_{B}(\mathcal{H}, \mathcal{K})}
\end{aligned}
$$

The expression for the dual norm follows by Proposition 1. Suppose now that $X \geq 0$, then by Corollary 3

$$
\begin{aligned}
\|X\|_{\mathcal{C}_{B}(\mathcal{H}, \mathcal{K})} & =\sup _{b \in B} \operatorname{Tr} X\left(I \otimes b^{\top}\right)=\inf _{Y \in \mathcal{C}_{B}(\mathcal{H}, \mathcal{K})} 2^{D_{\max }(X \| Y)} \\
\|X\|_{I \otimes B^{\top}} & =\sup _{Y \in \mathcal{C}_{B}(\mathcal{H}, \mathcal{K})} \operatorname{Tr} X Y=\inf _{b \in B} 2^{D_{\max }\left(X \| I \otimes b^{\top}\right)}
\end{aligned}
$$

By (11), $\operatorname{Tr} X\left(I \otimes b^{\top}\right)=\operatorname{Tr} \operatorname{Tr}{ }_{\mathcal{H}} X\left(I \otimes b^{\top}\right)=\operatorname{Tr} \Phi(b)$. Moreover, let $Y \in \mathcal{C}_{B}(\mathcal{H}, \mathcal{K})$ and let $S$ be the corresponding $B$-channel, then

$$
\operatorname{Tr} X Y=\operatorname{Tr} X(S \otimes i d)(\Psi)=\operatorname{Tr}\left(S^{*} \otimes i d\right)(X) \Psi=\left\langle\psi, X_{S^{*} \circ \Phi} \psi\right\rangle .
$$

\subsection{Channels}

Let $B=\mathfrak{S}(\mathcal{H})$, then generalized channels are the usual channels. In this case, we denote $\mathcal{C}_{B}(\mathcal{H}, \mathcal{K})$ by $\mathcal{C}(\mathcal{H}, \mathcal{K})$. Note that $\tilde{B}=\{I\}$ and $\mathcal{C}_{\tilde{B}}(\mathcal{H}, \mathcal{K})=\mathfrak{S}(\mathcal{K} \otimes \mathcal{H})$.

By Proposition 1, $\mathcal{C}(\mathcal{H}, \mathcal{K})$ is a faithful section of a base of $B(\mathcal{K} \otimes \mathcal{H})^{+}$and

$$
\widetilde{\mathcal{C}(\mathcal{H}, \mathcal{K})}=\left\{I_{\mathcal{K}} \otimes \rho, \rho \in \mathfrak{S}(\mathcal{H})\right\} .
$$

Furthemore, let $X \in B_{h}(\mathcal{K} \otimes \mathcal{H})$ and let $\Phi: B(\mathcal{H}) \rightarrow B(\mathcal{K})$ be the corresponding Hermitian map. Then by Theorem 2 .

$$
\|X\|_{\mathcal{C}(\mathcal{H}, \mathcal{K})}=\sup _{\sigma \in \mathfrak{S}(\mathcal{H} \otimes \mathcal{L})}\left\|\left(\Phi \otimes i d_{\mathcal{L}}\right)(\sigma)\right\|_{1}=\|\Phi\|_{\diamond}
$$

with $\operatorname{dim}(\mathcal{L})=\operatorname{dim}(\mathcal{H})$. For the dual norm, we have

$$
\|X\|_{I \otimes \mathfrak{S}(\mathcal{H})}=\inf _{\rho \in \mathfrak{S}(\mathcal{H})} \inf \{\lambda>0,-\lambda(I \otimes \rho) \leq X \leq \lambda(I \otimes \rho)\}
$$

If $\sigma \in B(\mathcal{K} \otimes \mathcal{H})^{+}$, we obtain

$$
\|\sigma\|_{I \otimes \mathfrak{S}(\mathcal{H})}=\inf _{\rho \in \mathfrak{S}(\mathcal{H})} 2^{D_{\max }(\sigma \| I \otimes \rho)}=2^{-H_{\min }(\mathcal{K} \mid \mathcal{H})_{\sigma}}
$$

where $H_{\min }(\mathcal{K} \mid \mathcal{H})_{\sigma}$ is the conditional min-entropy, see [18]. 


\subsection{Quantum supermaps}

Let $\mathcal{H}_{0}, \mathcal{H}_{1}, \ldots$ be a sequence of finite dimensional Hilbert spaces. For each $n \geq 1$, we define the sets $\mathcal{C}\left(\mathcal{H}_{0}, \ldots, \mathcal{H}_{n}\right)$ as follows: $\mathcal{C}\left(\mathcal{H}_{0}, \mathcal{H}_{1}\right)$ is, as before, the set of Choi matrices of channels $B\left(\mathcal{H}_{0}\right) \rightarrow B\left(\mathcal{H}_{1}\right)$. For $n>1$, we define $\mathcal{C}\left(\mathcal{H}_{0}, \ldots, \mathcal{H}_{n}\right)$ as the set of Choi matrices of cp maps $B\left(\mathcal{H}_{n-1} \otimes \cdots \otimes \mathcal{H}_{0}\right) \rightarrow$ $B\left(\mathcal{H}_{n}\right)$ that map $\mathcal{C}\left(\mathcal{H}_{0}, \ldots, \mathcal{H}_{n-1}\right)$ into $\mathfrak{S}\left(\mathcal{H}_{n}\right)$. Such maps were called quantum supermaps in $[13]^{2}$ and it was proved that for $n=2 N-1$ we get precisely the set of deterministic quantum $N$-combs for the sequence $\left\{\mathcal{H}_{0}, \ldots, \mathcal{H}_{2 N-1}\right\}$. If $n=2 N$, we get the set of $N+1$-combs for $\left\{\mathbb{C}, \mathcal{H}_{0}, \ldots, \mathcal{H}_{2 N}\right\}$.

Let us fix the sequence $\mathcal{H}_{0}, \mathcal{H}_{1}, \ldots$ and for this, put $\mathcal{C}_{n}=\mathcal{C}\left(\mathcal{H}_{0}, \ldots, \mathcal{H}_{n}\right)$. By using repeatedly Proposition 1, we see that $\mathcal{C}_{n}$ is a faithful section of a base of $B\left(\mathcal{H}_{n} \otimes \cdots \otimes \mathcal{H}_{0}\right)^{+}$and

$$
\mathcal{C}_{n+1}=\mathcal{C}_{\mathcal{C}_{n}}\left(\mathcal{H}_{n} \otimes \cdots \otimes \mathcal{H}_{0}, \mathcal{H}_{n+1}\right) .
$$

Moreover, by Proposition 1 .

$$
\widetilde{\mathcal{C}_{n}}=I_{\mathcal{H}_{n}} \otimes \mathcal{C}_{n-1}=\mathcal{C}\left(\mathcal{H}_{0}, \ldots, \mathcal{H}_{n}, \mathbb{C}\right)
$$

(note that $\mathcal{C}_{n-1}^{\top}=\mathcal{C}_{n-1}$, the last equality above follows from (11)). For $n=$ $2 N-1$, this corresponds to the set of $N$-round nonmeasuring co-strategies of [8, 9. Note also that for any finite dimensional Hilbert space $\mathcal{L}^{\prime}$,

$$
\begin{aligned}
\mathcal{C}_{\tilde{\mathcal{C}}_{n}}\left(\mathcal{H}_{n} \otimes \cdots \otimes \mathcal{H}_{0}, \mathcal{L}^{\prime}\right) & =\left\{Y \geq 0, \operatorname{Tr}_{\mathcal{L}^{\prime}} Y \in \mathcal{C}_{n}=\mathcal{C}_{\mathcal{C}_{n-1}}\left(\mathcal{H}_{n-1} \otimes \cdots \otimes \mathcal{H}_{0}, \mathcal{H}_{n}\right)\right\} \\
& =\left\{Y \geq 0, \operatorname{Tr}_{\mathcal{H}_{n}}\left(\operatorname{Tr}_{\mathcal{L}^{\prime}} Y\right) \in \widetilde{\mathcal{C}_{n-1}}\right\} \\
& =\mathcal{C}\left(\mathcal{H}_{0}, \ldots, \mathcal{H}_{n} \otimes \mathcal{L}^{\prime}\right)
\end{aligned}
$$

Now we obtain the following expressions for the corresponding norm and its dual.

Theorem 3. Let $n \geq 2$. Let $X \in B_{h}\left(\mathcal{H}_{n} \otimes \cdots \otimes \mathcal{H}_{0}\right)$ and let $\Phi: B\left(\mathcal{H}_{n-1} \otimes\right.$ $\left.\cdots \otimes \mathcal{H}_{0}\right) \rightarrow B\left(\mathcal{H}_{n}\right)$ be the corresponding map. We have

$$
\begin{aligned}
\|X\|_{\mathcal{C}\left(\mathcal{H}_{0}, \ldots, \mathcal{H}_{n}\right)} & =\sup _{Y_{1}, Y_{2} \geq 0, Y_{1}+Y_{2} \in \mathcal{C}\left(\mathcal{H}_{0}, \ldots, \mathcal{H}_{n}, \mathbb{C}\right)} \operatorname{Tr} X\left(Y_{1}-Y_{2}\right) \\
& =\sup _{Y \in \mathcal{C}\left(\mathcal{H}_{0}, \ldots, \mathcal{H}_{n}, \mathbb{C}\right)}\left\|Y^{1 / 2} X Y^{1 / 2}\right\|_{1} \\
& =\inf _{Y \in \mathcal{C}\left(\mathcal{H}_{0}, \ldots, \mathcal{H}_{n}\right)} \inf \{\lambda>0,-\lambda Y \leq X \leq \lambda Y\} \\
& =\sup _{\operatorname{dim}\left(\mathcal{L}^{\prime}\right)<\infty} \sup _{Y \in \mathcal{C}\left(\mathcal{H}_{0}, \ldots, \mathcal{H}_{n-2}, \mathcal{H}_{n-1} \otimes \mathcal{L}^{\prime}\right)}\left\|\left(\Phi \otimes i d_{\mathcal{L}^{\prime}}\right)(Y)\right\|_{1} \\
& \sup _{Y \in \mathcal{C}\left(\mathcal{H}_{0}, \ldots, \mathcal{H}_{n-2}, \mathcal{H}_{n-1} \otimes \mathcal{L}\right)}\left\|\left(\Phi \otimes i d_{\mathcal{L}}\right)(Y)\right\|_{1}
\end{aligned}
$$

where $\operatorname{dim}(\mathcal{L})=\operatorname{dim}\left(\mathcal{H}_{n-1} \otimes \cdots \otimes \mathcal{H}_{0}\right)$. Moreover, the dual norm is

$$
\|X\|_{I_{\mathcal{H}_{n}} \otimes \mathcal{C}\left(\mathcal{H}_{0}, \ldots, \mathcal{H}_{n-1}\right)}=\|X\|_{\mathcal{C}\left(\mathcal{H}_{0}, \ldots, \mathcal{H}_{n}, \mathbb{C}\right)}
$$

\footnotetext{
[5]

${ }^{2}$ Note that this definition is slightly different from the notion of supermap introduced in
} 
Proof. Duality of the norms is obtained from Theorem 2, this also implies the first equality. Next two equalities follow by Corollary 2 The rest follows by Theorem 2 .

For $n=2 N-1$, first two expressions are exactly the $N \diamond$-norm as obtained in [9] and [3. Duality of the norms corresponding to strategies and co-strategies was also obtained in $[9]$.

\section{A general quantum decision theory}

As before, let $B$ be a faithful section of a base of $B(\mathcal{H})^{+}, J=\operatorname{span}(B)$ and $Q=J \cap B(\mathcal{H})^{+}$. As we have seen, elements of $B$ may represent certain quantum devices and it is therefore reasonable to consider the following definitions.

Let $\left\{b_{\theta}, \theta \in \Theta\right\} \subset B$ be a parametrized family, for simplicity, we will suppose that the set of parameters $\Theta$ is finite. If $B$ is the set of states, the couple $\mathcal{E}=\left(\mathcal{H},\left\{b_{\theta}, \theta \in \Theta\right\}\right)$ is called an experiment and is interpreted as an a priori information on the true state of the system. Accordingly, for a section $B$, we will define a generalized experiment as a triple $\mathcal{E}=\left(\mathcal{H}, B,\left\{b_{\theta}, \theta \in \Theta\right\}\right)$.

Another ingredient of decision theory is a (finite) set $D$, the set of possible decisions. A decision procedure $\mathbf{m}$ is a procedure by which we pick some decision $d \in D$, with probability based on the "true value" of $b$. That is, $\mathbf{m}$ is a map $B \rightarrow \mathcal{P}(D)$, where $\mathcal{P}(D)$ is the set of probability measures on $D$, such a map will be called a measurement on $B$, with values in $D$. The payoff obtained if $d \in D$ is chosen while the true value is $\theta \in \Theta$ is given by the payoff function $w: \Theta \times D \rightarrow[0,1]$, the pair $(D, w)$ is called a (classical) decision problem. Let $\lambda$ be an a priori probability distribution on $\Theta$. The task is to maximize the average payoff, that is the value of

$$
\mathcal{L}_{\mathcal{E}, \lambda, w}(\mathbf{m}):=\sum_{\theta, d} \lambda_{\theta} w(\theta, d) \mathbf{m}\left(b_{\theta}\right)_{d}
$$

over all measurements $\mathbf{m}: B \rightarrow \mathcal{P}(D)$.

It is quite clear that any measurement $\mathbf{m}$ on $B$ is given by a collection $\left\{\mathbf{m}_{d}, d \in D\right\}$ of elements in $Q^{*}$ such that $\mathbf{m}(b)_{d}=\left\langle\mathbf{m}_{d}, b\right\rangle$ and that we must have $\sum_{d} \mathbf{m}_{d}=e_{B}$. Similarly as it was shown in [13], any measurement is given by a collection $\left\{M_{d}, d \in D\right\} \subset B(\mathcal{H})^{+}$such that $\mathbf{m}_{d}=\pi\left(M_{d}\right)$ and $\pi\left(\sum_{d} M_{d}\right)=e_{B}$, that is

$$
\sum_{d} M_{d} \in \pi^{-1}\left(e_{B}\right) \cap B(\mathcal{H})^{+}=\tilde{B} .
$$

Any such collection of positive operators will be called a generalized POVM (with respect to $B$ ), or a $B$-POVM. It is also clear that any $B$-POVM defines a measurement on $B$ (but it may happen that different generalized POVMs define the same measurement, see [13]). If $B=\mathfrak{S}(\mathcal{H})$, we obtain a (usual) positive operator valued measure $(\mathrm{POVM}) M=\left\{M_{d}, d \in D\right\} \subset B(\mathcal{H})^{+}, \sum_{d} M_{d}=I$. 
Let us denote by $\mathcal{M}_{B}(\mathcal{H}, D)$ the set of all generalized POVMs with respect to $B$ with values in $D$ and let $\left\{M_{d}, d \in D\right\} \in \mathcal{M}_{B}(\mathcal{H}, D)$. Let us denote

$$
M=\sum_{d \in D}|d\rangle\langle d| \otimes M_{d}^{\top} \in B\left(\mathcal{H}_{D} \otimes \mathcal{H}\right)^{+},
$$

where $\mathcal{H}_{D}$ is a Hilbert space with $\operatorname{dim}\left(\mathcal{H}_{D}\right)=|D|$ and $\{|d\rangle, d \in D\}$ an ONB in $\mathcal{H}_{D}$. Then it is clear that $M$ is a block-diagonal element in $\mathcal{C}_{B}\left(\mathcal{H}, \mathcal{H}_{D}\right)$. Conversely, it is clear that if $X=\sum_{d}|d\rangle\langle d| \otimes X_{d} \in \mathcal{C}_{B}\left(\mathcal{H}, \mathcal{H}_{D}\right)$, then $\left\{X_{d}^{\top}, d \in\right.$ $D\} \in \mathcal{M}_{B}(\mathcal{H}, D)$. In this way, we identify $\mathcal{M}_{B}(\mathcal{H}, D)$ with the subset of blockdiagonal elements in $\mathcal{C}_{B}\left(\mathcal{H}, \mathcal{H}_{D}\right)$.

Let now $(D, w)$ be a decision problem and let $\mathbf{m}$ be a decision procedure with corresponding $B$-POVM $M$. Then the average payoff is computed as

$$
\mathcal{L}_{\mathcal{E}, \lambda, w}(\mathbf{m})=\mathcal{L}_{\mathcal{E}, \lambda, w}(M):=\sum_{\theta, d} \lambda_{\theta} w(\theta, d) \operatorname{Tr} M_{d} b_{\theta}=\operatorname{Tr} \xi_{\mathcal{E}, \lambda, w} M^{\top}
$$

where

$\xi_{\mathcal{E}, \lambda, w}=\sum_{\theta} \sum_{d} \lambda_{\theta} w(\theta, d)|d\rangle\left\langle d\left|\otimes b_{\theta}=\sum_{d}\right| d\right\rangle\langle d| \otimes \sum_{\theta} \lambda_{\theta} w(\theta, d) b_{\theta} \in B\left(\mathcal{H}_{D} \otimes \mathcal{H}\right)^{+}$.

More generally, let $\mathcal{D}$ be a Hilbert space, $\operatorname{dim}(\mathcal{D})=k$ and let $W$ be a function $W: \theta \mapsto W_{\theta} \in B(\mathcal{D})^{+}$, with $W_{\theta} \leq I$. We call the pair $(\mathcal{D}, W)$ a quantum decision problem, [16. A decision procedure is now a $B$-channel $\Phi: B(\mathcal{H}) \rightarrow B(\mathcal{D})$ and the average payoff of $\Phi$ is given by

$$
\mathcal{L}_{\mathcal{E}, \lambda, W}(\Phi)=\sum_{\theta} \lambda_{\theta} \operatorname{Tr} \Phi\left(b_{\theta}\right) W_{\theta}
$$

If $X \in \mathcal{C}_{B}(\mathcal{H}, \mathcal{D})$ is the Choi matrix of $\Phi$, then the average payoff has the form

$$
\begin{aligned}
\mathcal{L}_{\mathcal{E}, \lambda, W}(\Phi)=\mathcal{L}_{\mathcal{E}, \lambda, W}(X) & :=\sum_{\theta} \lambda_{\theta} \operatorname{Tr}\left(W_{\theta} \operatorname{Tr}{ }_{\mathcal{H}}\left[\left(I_{\mathcal{D}} \otimes b_{\theta}^{\top}\right) X\right]\right) \\
& =\sum_{\theta} \operatorname{Tr}\left(\lambda_{\theta} W_{\theta} \otimes b_{\theta}^{\top}\right) X=\operatorname{Tr} \xi_{\mathcal{E}, \lambda, W} X^{\top},
\end{aligned}
$$

where

$$
\xi_{\mathcal{E}, \lambda, W}=\sum_{\theta} \lambda_{\theta} W_{\theta}^{\top} \otimes b_{\theta} \in B(\mathcal{D} \otimes \mathcal{H})^{+} .
$$

It is easy to see that the set of quantum decision problems contains also classical ones: Let $(D, w)$ be a classical decision problem and let $\mathcal{H}_{D}$ be as before. Let $W_{\theta}:=\sum_{d \in D} w(\theta, d)|d\rangle\langle d|$, then $\left(\mathcal{H}_{D}, W\right)$ is a quantum decision problem and $\xi_{\mathcal{E}, \lambda, W}=\xi_{\mathcal{E}, \lambda, w}$. Let $X \in \mathcal{C}_{B}\left(\mathcal{H}, \mathcal{H}_{D}\right)$ and $X=\sum_{c, d \in D}|c\rangle\langle d| \otimes X_{c d}$ $X_{c d} \in B(\mathcal{H})$. Since $\xi_{\mathcal{E}, \lambda, w}$ is block-diagonal, we have

$$
\mathcal{L}_{\mathcal{E}, \lambda, W}(X)=\mathcal{L}_{\mathcal{E}, \lambda, w}(M),
$$


where $M=\sum_{d}|d\rangle\langle d| \otimes X_{d d}$ is a $B$-POVM. In other words, for a classical decision problem one cannot get better results by considering quantum decision procedures. Conversely, let $(\mathcal{D}, W)$ be a quantum decision problem such that all the operators $W_{\theta}$ commute. Then there is a basis of $\mathcal{D}$ with respect to which all the operators $W_{\theta}$ are given by diagonal matrices, and the problem is equivalent to a classical problem, in the sense that the we obtain the same average payoffs. Hence we can view the set of classical decision problems as the subset of quantum decision problems such that the payoff function $W$ has commutative range.

Theorem 4. Let $\mathcal{E}=\left(\mathcal{H}, B,\left\{b_{\theta}, \theta \in \Theta\right\}\right)$ be a generalized experiment and let $(\mathcal{D}, W)$ be a quantum decision problem. Then the maximal average payoff is given by

$$
\mathcal{L}_{\mathcal{E}, \lambda, W}:=\max _{X \in \mathcal{C}_{B}(\mathcal{H}, \mathcal{D})} \mathcal{L}_{\mathcal{E}, \lambda, W}(X)=\left\|\xi_{\mathcal{E}, \lambda, W}\right\|_{I_{\mathcal{D}} \otimes B}
$$

If $(\mathcal{D}, W)$ is classical, then

$$
\mathcal{L}_{\mathcal{E}, \lambda, W}=\inf _{b \in B} \sup _{d \in D} 2^{D_{\max }\left(\sum_{\theta} \lambda_{\theta} w(\theta, d) b_{\theta} \| b\right)}
$$

Proof. By (14), the maximal average payoff is given by

$$
\mathcal{L}_{\mathcal{E}, \lambda, W}=\max _{X \in \mathcal{C}_{B}(\mathcal{H}, \mathcal{D})} \operatorname{Tr} \xi_{\mathcal{E}, \lambda, W} X^{\top}=\left\|\xi_{\mathcal{E}, \lambda, W}\right\|_{I_{\mathcal{D}} \otimes B}
$$

the last equality follows by Corollary 3 and Proposition 1 If $(\mathcal{D}, W)$ is classi$\mathrm{cal}$, then we may suppose that the matrices $W_{\theta}$ are diagonal. Then $\xi_{\mathcal{E}, \lambda, W}=$ $\sum_{d}|d\rangle\langle d| \otimes \xi_{\mathcal{E}, \lambda, W, d}$ is block-diagonal, where $\xi_{\mathcal{E}, \lambda, W, d}=\sum_{\theta} \lambda_{\theta} w(\theta, d) b_{\theta}$. By Corollary [3, and definition of $D_{\max }$,

$$
\begin{aligned}
\left\|\xi_{\mathcal{E}, \lambda, W}\right\|_{I_{\mathcal{D}} \otimes B} & =\inf _{b \in B} 2^{D_{\max }\left(\xi_{\mathcal{E}, \lambda, W} \| I_{\mathcal{D}} \otimes b\right)}=\inf _{b \in B} \inf \left\{\gamma>0, \xi_{\mathcal{E}, \lambda, W, d} \leq 2^{\gamma} b, \forall d \in D\right\} \\
& =\inf _{b \in B} \sup _{d \in D} 2^{D_{\max }\left(\xi_{\mathcal{E}, \lambda, W, d} \| b\right)}
\end{aligned}
$$

We can also use Corollary 4 to characterize decision procedures that maximize average payoff, we will call such procedures optimal with respect to $(\mathcal{E}, \lambda, W)$.

Corollary 5. Let $(\mathcal{D}, W)$ be a decision problem and let $X \in \mathcal{C}_{B}(\mathcal{H}, \mathcal{D})$. Then $X$ is optimal with respect to $(\mathcal{E}, \lambda, W)$ if and only if there is some element $q \in Q$ such that $\xi_{\mathcal{E}, \lambda, W} \leq I_{\mathcal{D}} \otimes q$ and

$$
\left((I \otimes q)-\xi_{\mathcal{E}, \lambda, W}\right) X^{\top}=0 .
$$

If $(\mathcal{D}, W)$ is classical, then a B-POVM $\left(M_{1}, \ldots, M_{\operatorname{dim}(\mathcal{D})}\right)$ is optimal if and only if there is some $q \in Q$ such that $\sum_{\theta} \lambda_{\theta} w(\theta, d) b_{\theta} \leq q$ for all $d$ and

$$
q \sum_{d} M_{d}=\sum_{\theta} \lambda_{\theta} b_{\theta} \sum_{d} w(\theta, d) M_{d}
$$


Proof. The first part follows directly by Theorem 4 and Corollary 4 . If $(\mathcal{D}, W)$ is classical, then $\xi_{\mathcal{E}, \lambda, W}$ is block-diagonal, so that $\xi_{\mathcal{E}, \lambda, W} \leq I \otimes q$ if and only if each block is majorized by $q$, that is $\sum_{\theta} \lambda_{\theta} w(\theta, d) b_{\theta} \leq q$. Moreover, (16) implies that

$$
\sum_{d} \operatorname{Tr}\left(q-\sum_{\theta} \lambda_{\theta} \rho_{\theta} w(\theta, d)\right) M_{d}=0 .
$$

Since this is a sum of nonnegative elements, it is zero if and only if each summand is equal to zero. Again by positivity, this is equivalent to (15).

In particular, in the case $B=\mathfrak{S}(\mathcal{H})$, we obtain the following optimality condition for POVMs.

Corollary 6. Let $\mathcal{E}=\left\{\sigma_{\theta}, \theta \in \Theta\right\}$ be an experiment and let $(D, w)$ be a classical decision problem. Then a POVM $\left\{M_{d}, d \in D\right\}$ is optimal with respect to $(\mathcal{E}, \lambda, W)$ if and only if $q:=\sum_{d} \sum_{\theta} \lambda_{\theta} \sigma_{\theta} w(\theta, d) M_{d}$ is hermitian and such that $\sum_{\theta} \lambda_{\theta} \sigma_{\theta} w(\theta, d) \leq q$ for all $d$.

Remark 1. Sometimes the function $W$ is interpreted as loss rather than payoff, then $\mathcal{L}_{\mathcal{E}, \lambda, W}(\Phi)$ is the average loss of the procedure $\Phi$ which has to be minimized. Let $W_{\theta}^{\prime}=I_{\mathcal{D}}-W_{\theta}$, then $\theta \mapsto W_{\theta}$ is again a payoff (or loss) function and we have

$$
\begin{gathered}
\min _{\Phi} \mathcal{L}_{\mathcal{E}, \lambda, W}=\min _{\Phi} \sum_{\theta} \lambda_{\theta} \operatorname{Tr} \Phi\left(b_{\theta}\right) W_{\theta}=\min _{\Phi} \sum_{\theta} \lambda_{\theta} \operatorname{Tr} \Phi\left(b_{\theta}\right)\left(I-W_{\theta}^{\prime}\right) \\
=1-\max _{\Phi} \mathcal{L}_{\mathcal{E}, \lambda, W^{\prime}}(\Phi)=1-\left\|\xi_{\mathcal{E}, \lambda, W^{\prime}}\right\|_{I_{\mathcal{D}} \otimes B}
\end{gathered}
$$

Moreover, an optimal procedure $\Phi$ that minimizes the loss is a maximizer for $\mathcal{L}_{\mathcal{E}, \lambda, W^{\prime}}$, hence satisfies the conditions of Corollary 5 , with $W$ replaced by $W^{\prime}$. Note that then the codtition from Corollary 6 is the same as obtained in [12].

Let $\left\{M_{d}, d \in D\right\}$ be a $B$-POVM with $\sum_{d} M_{d}=c \in \tilde{B}$. Then since $0 \leq$ $M_{d} \leq c$ for all $d$, we have

$$
M_{d}=c^{1 / 2} \Lambda_{d} c^{1 / 2}, \quad d \in D,
$$

where $\Lambda_{d}:=c^{-1 / 2} M_{d} c^{-1 / 2}$ defines a (usual) POVM on the support $\operatorname{supp} c$ of $c$. It follows that $\operatorname{Tr} x M_{d}=\operatorname{Tr} c^{1 / 2} x c^{1 / 2} \Lambda_{d}$, that is, we can decompose the measurement defined by $\left\{M_{d}\right\}$ into a cp map $\chi_{c}: x \mapsto c^{1 / 2} x c^{1 / 2}$ followed by the usual measurement given by $\left\{\Lambda_{d}\right\}$, note that $\chi_{c} \in \mathcal{C}_{B}(\mathcal{H}, \operatorname{supp} c)$ so that $\chi_{c}$ maps a generalized experiment $\mathcal{E}=\left(\mathcal{H}, B,\left\{b_{\theta}, \theta \in \Theta\right\}\right)$ onto an ordinary experiment $\mathcal{E}_{c}:=\left\{\operatorname{supp} c, \mathfrak{S}(\operatorname{supp} c),\left\{\chi_{c}\left(b_{\theta}\right), \theta \in \Theta\right\}\right)$. We write this decomposition as $M=\Lambda \circ \chi_{c}$. Such a decoposition was also used in [3] in the case of testers and in [13] for generalized POVMs. Using this decomposition, we obtain the following optimality condition for $B$-POVMs.

Corollary 7. Let $(D, w)$ be a classical decision problem and let $M \in \mathcal{M}_{B}(\mathcal{H}, D)$ with decomposition $M=\Lambda \circ \chi_{c}$. Suppose $c$ is invertible and let $\mathcal{E}_{c}:=\left(\mathcal{H},\left\{\sigma_{\theta}:=\right.\right.$ 
$\left.\left.\chi_{c}\left(b_{\theta}\right), \theta \in \Theta\right\}\right)$. Then $M$ is optimal for $(\mathcal{E}, \lambda, w)$ if and only if $\Lambda$ is optimal for $\left(\mathcal{E}_{c}, \lambda, w\right)$ and

$$
\sum_{d} \sum_{\theta} \lambda_{\theta} w(\theta, d) \sigma_{\theta} \Lambda_{d} \in \chi_{c}(J)
$$

Proof. Directly by Corollary 5 and 6 .

Example 2 (Multiple hypothesis testing). Suppose a family $\left\{b_{1}, \ldots, b_{k}\right\}$ of elements in $B$ is given and the task is to decide which is the true one, moreover, given some $\lambda \in \mathcal{P}(\{1, \ldots k\})$, we want to minimize the average probability of making an error. In this case, put $\mathcal{E}=\left(\mathcal{H}, B,\left\{b_{1}, \ldots, b_{k}\right\}\right), \Theta=D=\{1, \ldots, k\}$ and the loss function is $w(i, j)=1-\delta_{i j}$, where $\delta$ is the Kronecker symbol. A decision procedure is a $B$-POVM $\left\{M_{1}, \ldots, M_{k}\right\}$, where $M_{i}$ corresponds to the choice $b_{i}$. Then the average loss is the average error probability

$$
\mathcal{L}_{\mathcal{E}, \lambda, w}(M)=\sum_{i, j} \lambda_{i}\left(1-\delta_{i j}\right) \operatorname{Tr} b_{i} M_{j}=\sum_{i \neq j} \lambda_{i} \operatorname{Tr} b_{i} M_{j} .
$$

We can use Remark 1 to compute the minimal average error probability $\Pi_{\lambda}^{B}\left(b_{1}, \ldots, b_{k}\right):=$ $\min _{M} \mathcal{L}_{\mathcal{E}, \lambda, w}(M)$. We obtain $\xi_{\mathcal{E}, \lambda, w^{\prime}}=\sum_{i}|i\rangle\langle i| \otimes \lambda_{i} b_{i}$, so that the minimal average error probability is

$$
\Pi_{\lambda}^{B}\left(b_{1}, \ldots, b_{k}\right)=1-\left\|\xi_{\lambda, w^{\prime}}\right\|_{I \otimes B}=1-\inf _{b \in B} \sup _{1 \leq i \leq k} 2^{D_{\max }\left(\lambda_{i} b_{i} \| b\right)}
$$

For $B=\mathfrak{S}(\mathcal{H})$, the last equality was obtained in [7, see also [18].

Let us now look at an optimal decision procedure. Let $\left\{M_{i}\right\}$ be a $B$-POVM with decomposition $M=\Lambda \circ \chi_{c}$ and let us suppose that $c=\sum_{i} M_{i}$ is positive definite. Let $\sigma_{i}=\chi_{c}\left(b_{i}\right)$ and $\mathcal{E}_{c}=\left(\mathcal{H}, \mathfrak{S}(\mathcal{H}),\left\{\sigma_{1}, \ldots, \sigma_{k}\right\}\right)$. Suppose that $\left\{\Lambda_{i}\right\}$ is optimal for $\left(\mathcal{E}_{c}, \lambda, w\right)$, this is equivalent to the fact that $\sum_{i} \lambda_{i} \sigma_{i} \Lambda_{i}=: p$ is a hermitian element that majorizes $\lambda_{i} \sigma_{i}$ for all $i$. By Remark 1 and Corollary 7. $\left\{M_{i}\right\}$ is then optimal for $(\mathcal{E}, \lambda, w)$ if and only if $p \in c^{1 / 2} J c^{1 / 2}$, note that $\sigma_{i} \in c^{1 / 2} J c^{1 / 2}$ for all $i$.

Example 3 (Hypothesis testing). Let $k=2$ in the previous example, then we obtain the hypothesis testing, or discrimination problem, considered at the end of Section 1.2, Here we have

$$
\||0\rangle\left\langle 0\left|\otimes s b_{0}+\right| 1\right\rangle\langle 1| \otimes t b_{1} \|_{I_{2} \otimes B}=\frac{1}{2}\left(\left\|s b_{0}-t b_{1}\right\|_{B}+s+t\right)
$$

for $s, t>0$, so that indeed, $1-\left\|\xi_{\mathcal{E}, \lambda, w^{\prime}}\right\|_{I_{2} \otimes B}=\frac{1}{2}\left(1-\left\|\lambda b_{0}-(1-\lambda) b_{1}\right\|_{B}\right)$ is the minimal Bayes error probability. Let $\left\{M_{0}, M_{1}\right\}$ be a $B$-POVM such that $c=M_{0}+M_{1}$ is positive definite and let $\sigma_{i}=\chi_{c}\left(b_{i}\right)$. Suppose $\lambda=1 / 2$ and let $\Lambda_{i}=c^{-1 / 2} M_{i} c^{-1 / 2}$ be a POVM which is optimal for $\left(\mathcal{E}_{c}, \lambda, w\right)$, then $\Lambda_{0}$ is the projection onto the support of $\left(\sigma_{0}-\sigma_{1}\right)_{+}$and $\sum_{i} \lambda_{i} \sigma_{i} \Lambda_{i}=\frac{1}{2}\left(\left(\sigma_{0}-\sigma_{1}\right)_{+}+\sigma_{1}\right)$. From the previous example, it is clear that $\left\{M_{0}, M_{1}\right\}$ is then an optimal test for $(\mathcal{E}, \lambda, w)$ if and only if any of (and therefore all of) $\left(\sigma_{0}-\sigma_{1}\right)_{+},\left(\sigma_{0}-\sigma_{1}\right)_{-}$, $\left|\sigma_{0}-\sigma_{1}\right|$ is an element in $c^{1 / 2} J c^{1 / 2}$. 
In particular, let $B=\mathcal{C}(\mathcal{H}, \mathcal{K})$. In this case, the $B$-POVMs are exactly the quantum 1-testers of [3, 22, see also [13. More precisely, the $B$-POVMs $M=\left\{M_{d}, d \in D\right\} \subset B(\mathcal{K} \otimes \mathcal{H})^{+}$satisfy $\sum_{d} M_{d}=I \otimes \sigma$ for some $\sigma \in \mathfrak{S}(\mathcal{H})$. Then the decomposition $M=\Lambda \circ \chi_{I \otimes \sigma}$ corresponds to an implementation of the tester $M$ by a triple $\left(\mathcal{H}_{A}, \rho, \Lambda\right)$, where $\mathcal{H}_{A}=\operatorname{supp}(\sigma), \rho=\chi_{I \otimes \sigma}(\Psi)$ is a pure state in $\mathfrak{S}\left(\mathcal{H} \otimes \mathcal{H}_{A}\right)$ and

$$
\operatorname{Tr} M_{d} X_{\Phi}=\operatorname{Tr} \Lambda_{d}\left(\left(\Phi \otimes i d_{A}\right)(\rho)\right)
$$

Note that $\sigma=\operatorname{dim}(\mathcal{H})^{-1} I$ is obtained in the case that the input state $\rho$ is maximally entangled. By the results of Example 3, we have the following:

Corollary 8. Let $b_{i}=X_{\Phi_{i}}$ be Choi matrices of the channels $\Phi_{0}, \Phi_{1}: B(\mathcal{H}) \rightarrow$ $B(\mathcal{K})$. Consider the problem of testing the hypothesis $\Phi_{0}$ against $\Phi_{1}$, with a priori probability $\lambda \in[0,1]$. Then there exists an optimal 1-tester with maximally entangled input state if and only if $\operatorname{Tr} \mathcal{K}\left|\lambda X_{\Phi_{0}}-(1-\lambda) X_{\Phi_{1}}\right|$ is a multiple of $I_{\mathcal{H}}$.

\section{References}

[1] M.D. Choi, Completely positive maps on complex matrices, Lin. Alg. Appl. 10 (1975), 285-290

[2] G. Chiribella, G. M. D'Ariano, P. Perinotti, Quantum circuit architecture, Phys. Rev. Lett. 101 (2008), 060401

[3] G. Chiribella, G. M. D'Ariano, and P. Perinotti, Memory effects in quantum channel discrimination, Phys. Rev. Lett. 101 , 180501 (2008).

[4] G. Chiribella, G. M. D'Ariano, P. Perinotti, Theoretical framework for quantum networks, Phys. Rev. A 80 (2009), 022339

[5] G. Chiribella, G. M. D'Ariano, P. Perinotti, Transforming quantum operations: quantum supermaps, Europhysics Letters 83 (2008), 30004

[6] N. Datta, Min- and max- relative entropies and a new entanglement monotone, IEEE Transactions on Information Theory 55 (2009), 2816-2826

[7] N. Datta, M. Mosonyi, Generalized relative entropies and the capacity of classical-quantum channels, J. Math. Phys. 50 (2009), 072104

[8] Gus Gutoski and John Watrous, Toward a general theory of quantum games. In Proceedings of the 39th ACM Symposium on Theory of Computing (STOC 2007), 565574, 2007, arXiv:quant-ph/0611234v2

[9] G. Gutoski, On a measure of distance for quantum strategies, J. Math. Phys. 53 (2012), 032202

[10] C. E. Helstrom, Quantum Detection and Estimation Theory, Academic Press, New York (1976)

[11] A. S. Holevo, Probabilistic and Statistical Aspects of Quantum Theory, North Holland, Amsterdam (1982) 
[12] A. Holevo, Statistical Structure of Quantum Theory, Springer-Verlag Berlin Heidelberg (2001)

[13] A. Jenčová, Generalized channels: channels for convex subsets of the state space, J. Math. Phys. 53, 012201 (2011), arXiv:1105.1899

[14] A. Jenčová, Extremal generalized quantum measurements, arXiv:1207.5420v2

[15] A. Kitaev, Quantum computations: algorithms and error correction. Russian Mathematical Surveys, 52 (1997), 11911249

[16] K. Matsumoto, A quantum version of randomization criterion, arXiv:1012.2650v6, (2012)

[17] V. Paulsen, Completely Bounded Maps and Operator Algebras, Cambridge University Press, 2003

[18] R. Koenig, R. Renner, C. Schaffner, The operational meaning of min- and max-entropy, IEEE Trans. Inf. Th. 55 (2009)

[19] T. Rockafellar, Convex Analysis, Princeton University Press, 1970 Princeton

[20] J. Watrous, Notes on super-operator norms induced by Schatten norms, Quantum Information and Computation, 5 (2005), 58-68, arxiv:quant-ph/0411077v1

[21] D. Reeb, M.J. Kastoryano, M.M. Wolf, Hilbert's projective metric in quantum information theory, J. Math. Phys. 52 (2011), 082201

[22] M. Ziman, Process POVM: A mathematical framework for the description of process tomography experiments, Phys. Rev. A 77, 062112 (2008) 